\title{
Ensemble Kalman filtering for non-linear likelihood models using kernel-shrinkage regression techniques
}

\author{
Jon Sætrom • Henning Omre
}

Received: 16 June 2010 / Accepted: 3 December 2010 / Published online: 19 March 2011

(C) The Author(s) 2011. This article is published with open access at Springerlink.com

\begin{abstract}
One of the major limitations of the classical ensemble Kalman filter (EnKF) is the assumption of a linear relationship between the state vector and the observed data. Thus, the classical EnKF algorithm can suffer from poor performance when considering highly non-linear and non-Gaussian likelihood models. In this paper, we have formulated the EnKF based on kernel-shrinkage regression techniques. This approach makes it possible to handle highly non-linear likelihood models efficiently. Moreover, a solution to the preimage problem, essential in previously suggested EnKF schemes based on kernel methods, is not required. Testing the suggested procedure on a simple, illustrative problem with a non-linear likelihood model, we were able to obtain good results when the classical EnKF failed.
\end{abstract}

Keywords Shrinkage regression - Kernel methods • Sequential data assimilation $\cdot$ Model selection

\section{Introduction}

In recent years, Bayesian methods have become attractive to use when considering geophysical inverse problems [40]. The ensemble Kalman filter (EnKF) is a Bayesian method that provides a solution to highly non-linear and high-dimensional spatiotemporal data assimilation problems $[1,8]$. The EnKF is defined in the

\footnotetext{
J. Sætrom $(\varangle) \cdot$ H. Omre

Department of Mathematical Sciences,

Norwegian University of Science and Technology,

Alfred Getz' vei 1, 7034 Trondheim, Norway

e-mail: jonsa@math.ntnu.no
}

spirit of the classical Kalman filter (KF) [21], that provides the analytical solution of the posterior probability distribution when the prior, forward and likelihood models are Gaussian and linear, termed the Gausslinear model.

Analytical tractability of the posterior distribution will be lost in a general model setting. Thus, we may apply techniques such as Markov chain Monte Carlo (McMC) or rejection sampling to generate realisations from the posterior distribution of interest [6]. For highdimensional problems, however, these methods tend to be computationally prohibitive.

The EnKF approach is based on the approximation that the output of the forward and likelihood models are jointly Gaussian, with unknown mean and covariance. Using an ensemble of independent realisations to estimate the model parameters empirically, ensures that the EnKF is consistent with the KF for Gausslinear models as the ensemble size tends to infinity $[8,25]$.

As shown in Anderson [2], we can equally formulate the classical EnKF updating scheme as a multivariate linear regression problem, where the Kalman gain matrix defines the unknown matrix of regression coefficients. Hence, the classical EnKF can have poor performance when considering highly non-linear likelihood models. Methods such as the randomised maximum likelihood filter (RMLF) [31] can improve on the EnKF updating scheme for non-linear likelihood models. However, the RMLF algorithm requires an optimisation step making the method more computationally demanding than the traditional EnKF. This is especially true when considering high dimensional data such as time-lapse seismic. In addition, it is unclear how to use the RMLF if the error term is not additive [27]. 
The classical EnKF tends to underestimate the prediction uncertainty for small ensemble sizes $[10,17$, $28,37,48]$. This can potentially lead to an ensemble collapsing into one single realisation. In a recent paper, Sætrom and Omre [46], reformulated the classical EnKF updating scheme using shrinkage regression techniques known from multivariate statistics. It is well known from statistical literature that the unbiased classical least squares estimator for the matrix of regression coefficients is not optimal in the presence of collinear data, and can lead to severe problems of model overfitting [12]. The purpose of shrinkage regression techniques is therefore to replace the classical, unbiased estimator of the unknown matrix of regression coefficients with biased alternatives having improved predictive capabilities; e.g. using dimension reduction techniques on the predictor variables, or by regularising the estimated matrix of regression coefficients. Because the updated ensemble members will be coupled through the estimated Kalman gain matrix, collinearities between the ensemble members will eventually occur [17]. Hence, it is not surprising that applying shrinkage regression techniques can lead to significant improvements compared with the classical EnKF updating scheme, as illustrated in the examples considered in Sætrom and Omre [46].

In recent years, kernel methods have become popular within the field of machine learning [44, 47]. The aim of these methods is to transform data from the original vector space into a possibly high dimensional feature space, where we assume that the underlying model assumptions, such as linear dependencies in a regression setting, are valid. Kernel methods are frequently used in non-linear principal component analysis (PCA) [39, 41], data mining [18], classification [49] and non-linear regression [36]. Common for these methods is that the algorithms can be reformulated through inner products in the original space. Because we can define inner products in a feature space through positive definite functions, known as kernel functions, there is no need to generate realisations in the feature space $[15,44]$.

In the current paper, we extend the EnKF updating scheme to a non-linear setting using previously defined kernel-based shrinkage regression techniques [36, 47]. We demonstrate the suggested approach on a simplistic example with a non-linear and non-Gaussian likelihood model. The procedure has the same computational complexity and memory requirements as the fastest implementations of the traditional EnKF.

We are not the first to recognise the potential of kernel methods in an EnKF setting [3, 38]. However, the focus of these studies is to incorporate highly nonGaussian features of the state vector into the EnKF, which require a solution to the pre-image problem of mapping the state vector from the feature space back to the original space. Solving this problem using traditional approaches requires non-linear optimisation techniques [24, 26, 44], which can lead to high computational demands. In the current study we use kernel methods for handling non-linearities in the likelihood model, which do not require this back-transformation.

\section{Notation and model formulation}

Throughout the paper, we use the notation $\boldsymbol{x} \in \mathbb{R}^{n_{x} \times 1}$ to denote that $\boldsymbol{x}$ is an $n_{x}$-dimensional column vector in the real space and $\boldsymbol{x}^{T}$ will denote its transpose. Similarly, we will write $\boldsymbol{X} \in \mathbb{R}^{m \times n}$ to denote that $\boldsymbol{X}$ is a matrix in the real space containing $m$ rows and $n$ columns. Note that we will use that the same notation for both scalars and random variables. Probability density functions (PDF) will be denoted by $f(\boldsymbol{x})$, and the notation $\boldsymbol{x} \sim f(\boldsymbol{x})$, implies that the random vector $\boldsymbol{x}$ follows the PDF $f(\boldsymbol{x})$. Furthermore, we will denote the conditional PDF of $\boldsymbol{x}$ given $\boldsymbol{y}$ by $f(\boldsymbol{x} \mid \boldsymbol{y})$. As a special case, the notation $\boldsymbol{x} \sim \operatorname{Gauss}_{n_{x}}\left(\boldsymbol{\mu}_{\boldsymbol{x}}, \boldsymbol{\Sigma}_{\boldsymbol{x}}\right)$ will be used to denote that $\boldsymbol{x}$ follows the $n_{x}$-dimensional multivariate Gaussian distribution with mean vector $\boldsymbol{\mu}_{\boldsymbol{x}}$ and covariance matrix $\boldsymbol{\Sigma}_{\boldsymbol{x}}$.

Consider the sequence of stochastic vectors $\boldsymbol{x}_{t_{0}}, \ldots$, $\boldsymbol{x}_{t_{K+1}} ; \boldsymbol{x}_{t_{i}} \in \mathbb{R}^{n_{x} \times 1}$ and $\boldsymbol{d}_{t_{0}}, \ldots, \boldsymbol{d}_{t_{K}} ; \boldsymbol{d}_{t_{i}} \in \mathbb{R}^{n_{d} \times 1}$, outlined in Fig. 1. Here, $\boldsymbol{x}_{t_{k}}$ denotes the state of the unknown random vector of interest at time step $k$ and time $t_{k}$, and similarly $\boldsymbol{d}_{t_{k}}$ denotes the vector of observed data. For notational convenience, we will from now on drop the subscript $t_{k}$, and simply write $\boldsymbol{x}_{k}$ and $\boldsymbol{d}_{k}$. Also note that we will for simplicity refer to $\boldsymbol{x}$ and $\boldsymbol{d}$ as the state and observation vector respectively.

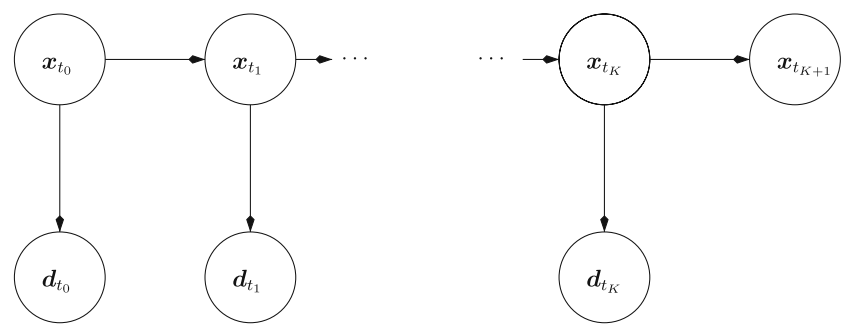

Fig. 1 Stochastic directed acyclic graph (DAG) of the model considered 
Let $f\left(x_{0}\right)$ denote the PDF of the state vector at the initial time step. The Markov property of the directed acyclic graph (DAG) in Fig. 1 entails

$f\left(\boldsymbol{x}_{k+1} \mid \boldsymbol{x}_{k}, \boldsymbol{x}_{k-1}, \ldots, \boldsymbol{x}_{0}\right)=f\left(\boldsymbol{x}_{k+1} \mid \boldsymbol{x}_{k}\right), k=0, \ldots, K$.

We define the PDF, $f\left(\boldsymbol{x}_{k+1} \mid \boldsymbol{x}_{k}\right)$, through a known, possibly highly non-linear forward function, $\omega:(\epsilon$ $\left.\mathbb{R}^{n_{x} \times 1} \times \in \mathbb{R}^{n_{x} \times 1}\right) \rightarrow \in \mathbb{R}^{n_{x} \times 1}$, which implies

$\boldsymbol{x}_{k+1}=\omega\left(\boldsymbol{x}_{k}, \boldsymbol{\epsilon}_{\boldsymbol{x}_{k}}\right), k=0, \ldots, K$.

Here, $\boldsymbol{\epsilon}_{\boldsymbol{x}_{k}} \in \mathbb{R}^{n_{x} \times 1}$ represents random model errors or numerical errors in the forward model, assumed to follow a known probability distribution. Thus, $f\left(\boldsymbol{x}_{0}\right)$ and $\boldsymbol{\omega}(\cdot, \cdot)$ implicitly defines the prior $\operatorname{PDF} f\left(\boldsymbol{x}_{0}, \ldots, \boldsymbol{x}_{K+1}\right)$. We define the likelihood function, $f\left(\boldsymbol{d}_{k} \mid \boldsymbol{x}_{k}\right)$, through a known, non-linear function $\zeta:\left(\mathbb{R}^{n_{x} \times 1} \times \mathbb{R}^{n_{d} \times 1}\right) \rightarrow$ $\mathbb{R}^{n_{d} \times 1}$, that is,

$\boldsymbol{d}_{k}=\zeta\left(\boldsymbol{x}_{k}, \boldsymbol{\epsilon}_{\boldsymbol{d}_{k}}\right), k=0, \ldots, K$.

Again, $\boldsymbol{\epsilon}_{\boldsymbol{d}_{k}} \in \mathbb{R}^{n_{d} \times 1}$, represents the random observation error following a known PDF.

For notational convenience, we from now on let

$\boldsymbol{x}_{k}^{c} \sim f\left(\boldsymbol{x}_{k} \mid \boldsymbol{d}_{0: k}\right)$

$\boldsymbol{x}_{k}^{u} \sim f\left(\boldsymbol{x}_{k} \mid \boldsymbol{d}_{0: k-1}\right), \quad k=1, \ldots, K+1$,

where $\boldsymbol{d}_{0: l}$ denotes the sequence $\boldsymbol{d}_{0}, \ldots, \boldsymbol{d}_{l}$ for $l>$ 0 . Bayesian inversion provides a sequential solution to the spatiotemporal forecast problem of predicting $\boldsymbol{x}_{k}^{c}$, for $k=1, \ldots, K+1$. With such an approach, we assess the unknown vectors $\boldsymbol{x}_{k}^{c}$ and $\boldsymbol{x}_{k+1}^{u}$ by sampling from the respective posterior distributions, $f\left(\boldsymbol{x}_{k} \mid \boldsymbol{d}_{0: k}\right)$ and $f\left(\boldsymbol{x}_{k+1} \mid \boldsymbol{d}_{0: k}\right)$. Using Bayes rule and the Markov property of the DAG in Fig. 1, which entails $f\left(\boldsymbol{d}_{k} \mid \boldsymbol{x}_{k}, \boldsymbol{d}_{0:(k-1)}\right)=f\left(\boldsymbol{d}_{k} \mid \boldsymbol{x}_{k}\right)$, for $k=1, \ldots, K$, we get

$f\left(\boldsymbol{x}_{k} \mid \boldsymbol{d}_{0: k}\right) \propto f\left(\boldsymbol{x}_{k} \mid \boldsymbol{d}_{0:(k-1)}\right) f\left(\boldsymbol{d}_{k} \mid \boldsymbol{x}_{k}\right)$

$f\left(\boldsymbol{x}_{k+1} \mid \boldsymbol{d}_{0: k}\right)=\int f\left(\boldsymbol{x}_{k+1} \mid \boldsymbol{x}_{k}\right) f\left(\boldsymbol{x}_{k} \mid \boldsymbol{d}_{0: k}\right) \mathrm{d} \boldsymbol{x}_{k}$.

Generally, we only know the conditional distributions defined in Eq. 3 up to an unknown normalising constant. One possibility is to use computationally demanding techniques such as McMC or rejection sampling to generate realisation from the correct posterior distribution [6]. However, for applications such as petroleum reservoir evaluation, these techniques are computationally prohibitive [8]. An approximate solution can be obtained by assuming that $\boldsymbol{x}_{k}^{u}$ and $\boldsymbol{d}_{k}$ follows a distribution that ensures analytical tractability of $f\left(\boldsymbol{x}_{k} \mid \boldsymbol{d}_{0: k}\right)$, such as the Gaussian. These model assumptions are equivalent to those made in the EnKF [7].

\section{Classical ensemble Kalman filter}

Let

$\boldsymbol{x}_{k}^{u(i)}=\omega\left(\boldsymbol{x}_{k-1}^{c(i)}, \boldsymbol{\epsilon}_{\boldsymbol{x}_{k-1}}^{(i)}\right)$

and

$\boldsymbol{d}_{k}^{(i)}=\zeta\left(\boldsymbol{x}_{k}^{u(i)}, \boldsymbol{\epsilon}_{\boldsymbol{d}_{k}}^{(i)}\right)$,

for $i=1, \ldots, n_{e}$, and define $\boldsymbol{X}_{k}=\left[\boldsymbol{x}_{k}^{u(1)}, \ldots, \boldsymbol{x}_{k}^{u\left(n_{e}\right)}\right] \in$ $\mathbb{R}^{n_{x} \times n_{e}}$ and $\boldsymbol{D}_{k}=\left[\boldsymbol{d}_{k}^{(1)}, \ldots, \boldsymbol{d}_{k}^{\left(n_{e}\right)}\right] \in \mathbb{R}^{n_{d} \times n_{e}}$ as the state ensemble and data ensembles matrices respectively. For notational convenience, we will from now on omit the subscript $k$ because the focus will be on a single time step.

If we assume that the joint distribution of $\left(\boldsymbol{x}^{u}, \boldsymbol{d}\right)$ is Gaussian, a classical updating scheme for each ensemble member would be:

$\boldsymbol{x}^{c(i)}=\boldsymbol{x}^{u(i)}+\hat{\boldsymbol{K}}\left(\boldsymbol{d}-\boldsymbol{d}^{(i)}\right)$,

where

$\hat{\boldsymbol{K}}=\boldsymbol{X} \boldsymbol{H} \boldsymbol{D}^{T}\left(\boldsymbol{D} \boldsymbol{H} \boldsymbol{D}^{T}\right)^{-1} \in \mathbb{R}^{n_{x} \times n_{d}}$.

We refer to this as the classical EnKF updating scheme, where we denote the estimated Kalman gain matrix by $\hat{\boldsymbol{K}}$. Here,

$\boldsymbol{H}=\boldsymbol{I}-\frac{1}{n_{e}} \mathbf{1 1}^{T} \in \mathbb{R}^{n_{e} \times n_{e}}$

is the idempotent centring matrix, where $\boldsymbol{I}$ is the identity matrix, and $\mathbf{1}$ is a vector with each entry equal to one, both having proper dimensions. Under the Gaussian assumption stated above, $\boldsymbol{x}_{k}^{c(i)}$ will tend towards a realisation from the Gaussian posterior distribution $f\left(\boldsymbol{x}_{k} \mid \boldsymbol{d}_{0: k}\right)$ as $n_{e} \rightarrow \infty$.

From multivariate statistical theory, we know that the estimated Kalman gain matrix is equal to the least squares estimate of the matrix of regression coefficients in a multivariate linear regression setting [42]:

$\hat{\boldsymbol{K}}=\arg \min _{\boldsymbol{K}} \operatorname{tr}\left\{(\boldsymbol{X} \boldsymbol{H}-\boldsymbol{K} \boldsymbol{D} \boldsymbol{H})(\boldsymbol{X} \boldsymbol{H}-\boldsymbol{K} \boldsymbol{D} \boldsymbol{H})^{T}\right\}$,

where $\operatorname{tr}\{\cdot\}$ denotes the trace operator. Hence, it is not surprising that the classical EnKF can perform poorly for highly non-linear functions in the likelihood model.

Note that for situations where $n_{d} \geq n_{e}$, the matrix $\boldsymbol{D} \boldsymbol{H} \boldsymbol{D}^{T}$ in Eq. 5 will be singular, meaning that its 
inverse does not exist. To avoid this problem we can add a positive definite matrix, $\left(n_{e}-1\right) \Sigma_{r}$, which corresponds to adding a regularisation term, $\left(n_{e}-\right.$ 1) $\operatorname{tr}\left\{\boldsymbol{K} \Sigma_{r} \boldsymbol{K}^{T}\right\}$, to the objective function in Eq. 7. This gives

$$
\hat{\boldsymbol{K}}=\boldsymbol{X} \boldsymbol{H} \boldsymbol{D}^{T}\left(\boldsymbol{D} \boldsymbol{H} \boldsymbol{D}^{T}+\left(n_{e}-1\right) \boldsymbol{\Sigma}_{r}\right)^{-1},
$$

which is an extension of the standard EnKF updating scheme to non-linear likelihood models, similar to the approach in [8, Appendix A.2]. Assuming the following likelihood model, $\boldsymbol{d}=\boldsymbol{\zeta}\left(\boldsymbol{x}^{u}\right)+\boldsymbol{\epsilon}_{\boldsymbol{d}}$, where the independent Gaussian noise term has zero mean and covariance, $\boldsymbol{\Sigma}_{r}$, the Kalman gain estimate defined in Eq. 8 is natural to consider. If we replace the data ensemble matrix used in Eq. 8, with an alternative data ensemble matrix, $\tilde{\boldsymbol{D}}=\left[\zeta\left(\boldsymbol{x}^{u(1)}\right), \ldots, \zeta\left(\boldsymbol{x}^{u\left(n_{e}\right)}\right)\right]$, the estimator

$$
\hat{\boldsymbol{K}}=\boldsymbol{X} \boldsymbol{H} \tilde{\boldsymbol{D}}^{T}\left(\tilde{\boldsymbol{D}} \boldsymbol{H} \tilde{\boldsymbol{D}}^{T}+\left(n_{e}-1\right) \boldsymbol{\Sigma}_{r}\right)^{-1},
$$

will indeed be consistent with the estimator defined in Eq. 5. This follows because the data ensemble matrix can be split into two parts, $\boldsymbol{D}=\tilde{\boldsymbol{D}}+\boldsymbol{E}$, where $\boldsymbol{E}=\left[\boldsymbol{\epsilon}_{\boldsymbol{d}}^{(1)}, \ldots, \boldsymbol{\epsilon}_{\boldsymbol{d}}^{\left(n_{e}\right)}\right]$ is error perturbation ensemble matrix. However, in the general model setting with $\boldsymbol{d}=$ $\boldsymbol{\zeta}\left(\boldsymbol{x}, \boldsymbol{\epsilon}_{\boldsymbol{d}}\right)$, the two estimators will not be consistent.

Another potential problem with the classical least squared estimate of the Kalman gain is model overfitting. This is especially true in the presence of collinear data [9]. Because we couple the updated ensemble members through the estimated Kalman gain matrix, they can become increasingly collinear with time [28]. This can potentially lead to an ensemble collapsing into a single realisation, and certainly lead to an underestimation of the prediction uncertainty [46].

Fortunately, we can handle the problems described above efficiently using kernel shrinkage regression techniques known from multivariate non-linear regression, which we will consider next.

\section{Kernel-shrinkage regression}

To motivate the use of kernel methods, we start this section with a probabilistic discussion of a nonlinear regression problem with multivariate predictor variables:

$x=\gamma(\boldsymbol{d})+\epsilon_{x \mid \boldsymbol{d}}$.

Here, $x$ is a univariate, centred response variable; $\boldsymbol{d}$ is a centred multivariate predictor variable; $\gamma(\boldsymbol{d})$ is the non-linear regression function; and $\epsilon_{x \mid d}$ is a univariate Gaussian error term having zero mean and variance $\sigma_{x \mid d}^{2}$. For notational simplicity we only consider one-dimensional response variables, because it can be shown [12] that we under certain assumptions obtain corresponding solutions for each component in the multivariate case.

Following Williams [50], let the non-linear function $\gamma(\cdot)$ be decomposed into $L$ terms

$x=\boldsymbol{\beta}^{T} \varphi(d)+\epsilon_{x \mid d}$,

where $\boldsymbol{\beta}=\left[\beta_{1}, \ldots, \beta_{L}\right]^{T} \sim \operatorname{Gauss}_{L}(\mathbf{0}, \boldsymbol{I})$ is an unknown random vector. Here $\mathbf{0}$ is the zero vector of proper dimensions, and the $L$-dimensional vector $\varphi(\boldsymbol{d})=\left[\varphi_{1}(\boldsymbol{d}), \ldots, \varphi_{L}(\boldsymbol{d})\right]^{T}$, is a collection of known link functions. Two examples of link functions are

$\varphi_{k}(\boldsymbol{d})=a_{0_{k}}+a_{1_{k}} \boldsymbol{d}_{k}+a_{2_{k}} \boldsymbol{d}_{k}^{2}$

$\varphi_{k}(\boldsymbol{d})=\sigma_{\varphi_{k}} \exp \left\{-\sum_{l=1}^{n_{d}} \frac{\left(d_{l}-\tau_{k, l}\right)^{2}}{\Delta_{k, l}^{2}}\right\}$,

$k=1, \ldots, L$, where $a_{i, k}, \sigma_{\varphi_{k}}, \tau_{k, l}$ and $\Delta_{k, l}$ are model parameters. Under the assumption of independent observation errors for $\epsilon_{x \mid d}$ this entails:

$\mathrm{E}[x]=0$

$\operatorname{Cov}\left(x, x^{\prime}\right)=\varphi(d)^{T} \varphi\left(d^{\prime}\right)+\delta\left(d, d^{\prime}\right) \sigma_{x \mid d}^{2}$,

with $\delta(\cdot, \cdot)$ being the Dirac function taking value one when the arguments are identical and zero otherwise.

Consider a set of centred realisations $\left\{\left(x^{(i)}, \boldsymbol{d}^{(i)}\right), i=\right.$ $\left.1, \ldots, n_{e}\right\}$ and define the corresponding univariate, centred state vector $\tilde{\boldsymbol{x}}=\left[x^{(1)}, \ldots, x^{\left(n_{e}\right)}\right] \in \mathbb{R}^{n_{e} \times 1}$ with associated centred data ensemble matrix $\boldsymbol{D}$. In addition, let $\boldsymbol{d}^{*}$ be a new, centred data vector, with unknown centred state variable $x^{*}$. Under the Gaussian assumption above we have

$\left[\begin{array}{c}x^{*} \\ \tilde{\boldsymbol{x}}\end{array}\right] \sim \operatorname{Gauss}_{1+n_{e}}\left(\left[\frac{0}{\mathbf{0}}\right],\left[\begin{array}{cc}\operatorname{Cov}\left(x^{*}\right) & \operatorname{Cov}\left(x^{*}, \tilde{\boldsymbol{x}}\right) \\ \operatorname{Cov}\left(\tilde{\boldsymbol{x}}, x^{*}\right) & \operatorname{Cov}(\tilde{\boldsymbol{x}})\end{array}\right]\right)$,

where $\operatorname{Cov}\left(x^{*}\right)=\boldsymbol{\varphi}\left(\boldsymbol{d}^{*}\right)^{T} \boldsymbol{\varphi}\left(\boldsymbol{d}^{*}\right)+\sigma_{x \mid \boldsymbol{d}}^{2}, \quad \operatorname{Cov}\left(x^{*}, \tilde{\boldsymbol{x}}\right)=$ $\boldsymbol{\varphi}\left(\boldsymbol{d}^{*}\right)^{T} \boldsymbol{\Phi}$ and $\operatorname{Cov}(\tilde{\boldsymbol{x}})=\boldsymbol{\Phi}^{T} \boldsymbol{\Phi}+\sigma_{x \mid d}^{2} \boldsymbol{I}$, with $\boldsymbol{\Phi}=$ $\left[\boldsymbol{\varphi}\left(\boldsymbol{d}^{(1)}\right), \ldots, \boldsymbol{\varphi}\left(\boldsymbol{d}^{\left(n_{e}\right)}\right)\right] \in \mathbb{R}^{L \times n_{e}}$. The conditional expectation of $x^{*}$ given $\tilde{\boldsymbol{x}}, \mathrm{E}\left[x^{*} \mid \tilde{\boldsymbol{x}}\right]$, minimises the mean squared prediction error (MSPE), $\mathrm{E}\left[\left(x^{*}-g(\tilde{\boldsymbol{x}})\right)^{2}\right]$, for any function $g: \mathbb{R}^{n_{e}} \rightarrow \mathbb{R}[32]$. Hence,

$\hat{x}^{*}=\tilde{\boldsymbol{x}}^{T}\left(\boldsymbol{\Phi}^{T} \boldsymbol{\Phi}+\sigma_{x \mid d}^{2} \boldsymbol{I}\right)^{-1} \boldsymbol{\Phi}^{T} \boldsymbol{\varphi}\left(\boldsymbol{d}^{*}\right)$

is the optimal predictor of $x^{*}$ in terms of the MSPE. 
An alternative formulation of the non-linear regression problem is to parametrise $\gamma(\boldsymbol{d})$ by a Gaussian random field (GRF), as defined in Appendix A, with mean $\mathrm{E}[\gamma(\boldsymbol{d})]=0$, and covariance function $\operatorname{Cov}\left(\gamma(\boldsymbol{d}), \gamma\left(\boldsymbol{d}^{\prime}\right)\right)=c\left(\boldsymbol{d}, \boldsymbol{d}^{\prime}\right)$. Using the definition of a GRF together with well-known results from multivariate Gaussian theory, the predictive mean of $x^{*}$ given $\tilde{\boldsymbol{x}}$ is:

$\mathrm{E}\left[x^{*} \mid \tilde{\boldsymbol{x}}\right]=\tilde{\boldsymbol{x}}^{T}\left(\boldsymbol{C}+\sigma_{x \mid \boldsymbol{d}}^{2} \boldsymbol{I}\right)^{-1} \boldsymbol{c}^{*}$

with $\boldsymbol{c}^{*}=\left[c\left(\boldsymbol{d}^{(1)}, \boldsymbol{d}^{*}\right), \ldots, c\left(\boldsymbol{d}^{\left(n_{e}\right)}, \boldsymbol{d}^{*}\right)\right]^{T} \in \mathbb{R}^{n_{e} \times 1}$, and

$\boldsymbol{C}=\left[\begin{array}{ccc}c\left(\boldsymbol{d}^{(1)}, \boldsymbol{d}^{(1)}\right) \ldots c\left(\boldsymbol{d}^{(1)}, \boldsymbol{d}^{\left(n_{e}\right)}\right) \\ \vdots & \ddots & \vdots \\ c\left(\boldsymbol{d}^{\left(n_{e}\right)}, \boldsymbol{d}^{(1)}\right) \ldots & c\left(\boldsymbol{d}^{\left(n_{e}\right)}, \boldsymbol{d}^{\left(n_{e}\right)}\right)\end{array}\right] \in \mathbb{R}^{n_{e} \times n_{e}}$.

Comparing Eqs. 12 and 13, we see that there is a dual formulation of the non-linear regression problem, where we can interpret inner products between vectors $\varphi(\cdot) \in \mathbb{R}^{L \times 1}$ as covariance functions $c(\cdot, \cdot)$ of a GRF. For the link-function defined in Eq. 11 this can be realised by letting $L \rightarrow \infty$ and selecting $\Delta_{k, l}=\Delta$ for all $k$ and $l$, which entails [11]:

$c\left(\boldsymbol{d}, \boldsymbol{d}^{\prime}\right)=\boldsymbol{\varphi}(\boldsymbol{d})^{T} \boldsymbol{\varphi}\left(\boldsymbol{d}^{\prime}\right)=\sigma_{\varphi}^{2} \exp \left\{-\frac{1}{2 \Delta}\left\|\boldsymbol{d}-\boldsymbol{d}^{\prime}\right\|_{2}^{2}\right\}$

which we recognise as the second-order exponential covariance function. The assumption that we can describe inner products between vectors $\varphi(d)$ and $\varphi\left(d^{\prime}\right)$ through symmetric, positive definite functions, $c\left(\boldsymbol{d}, \boldsymbol{d}^{\prime}\right)$, is the foundation of kernel methods known from the machine learning literature [44].

\subsection{Kernel methods}

We now generalise the mapping $\varphi(\cdot)$, and let $\varphi: \mathbb{R}^{n_{d}} \rightarrow$ $\mathcal{F}$, where $\mathcal{F}$ is some unspecified inner product space [52], which we for simplicity will refer to as the feature space. In the non-linear regression setting this entails transforming the predictor variable, $\boldsymbol{d}$, into a feature space where the linear relationship with the response variable, $x$, is valid, as described in Fig. 2.

In Eq. 12, we express $\hat{x}^{*}$ through inner products in the feature space, which we can equally describe through values of the covariance function, $c(\cdot, \cdot)$, under the GRF assumption. In general, this will hold for any symmetric, positive definite function, $c(\cdot, \cdot)$, also known as kernel functions [44]. In the machine learning literature, this is known as the "kernel-trick" [44], which in practise means that we can reformulate any algorithm involving inner products in the input
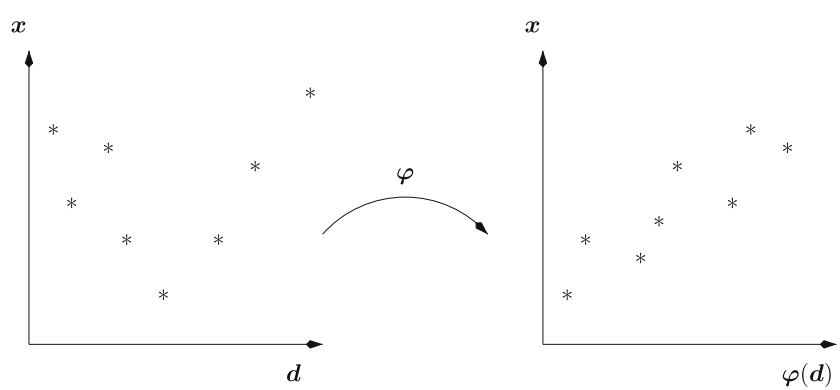

Fig. 2 Transformation of $\boldsymbol{d}$ into a feature space $\mathcal{F}$, where there is a linear relationship with the response $\boldsymbol{x}$

space (here $\mathbb{R}^{n_{d}}$ ) into a feature space, $\mathcal{F}$, using kernel functions. The formal conditions for which the assumption, $c\left(\boldsymbol{d}, \boldsymbol{d}^{\prime}\right)=\left\langle\boldsymbol{\varphi}(\boldsymbol{d}), \boldsymbol{\varphi}\left(\boldsymbol{d}^{\prime}\right)\right\rangle$ holds are described in Hoffmann et al. [15], namely symmetry and positive definiteness. The interested reader can find further details in Smola and Schölkopf [44] or Taylor and Cristianini [47].

Sætrom and Omre [46], reformulated the classical EnKF updating scheme using principal component regression (PCR) [16] and partial least squares regression (PLSR) [51], known from shrinkage regression. Both methods can be transformed into a non-linear setting using kernelised versions; see Rosipal et al. [35] and Rosipal and Trejo [36] for PCR and PLSR, respectively. Common to the kernel versions of these two shrinkage regression techniques is that we perform a dimension reduction in the feature space. Moreover, given a new observation vector, $\boldsymbol{d}^{*}$, we predict the state variable, $x^{*}$, by evaluating the kernel function. Hence, the methods does not require an inverse mapping, avoiding the preimage problem occurring when using the kernel approach on the state variables $[3,38]$.

\subsection{Comments}

An early reference to the Bayesian formulation of the non-linear regression problem is O'Hagan [30]. However, the connection with kernel methods known from the machine learning literature was not realised until recently $[33,50]$. Moreover, readers familiar with Geostatistics will surely recognise the connection between the probabilistic formulation above and a generalisation of kriging using non-stationary covariance models $[5,19]$.

The decoupling of the state vector into univariate components, as done in the decomposition above, is theoretically only valid if the elements in the stochastic error term, $\boldsymbol{\epsilon}_{\boldsymbol{x} \mid \boldsymbol{d}}$, are independent, which implies that the 
covariance $\boldsymbol{\Sigma}_{\boldsymbol{\epsilon}_{\mathrm{x} \mid d}}$ is proportional to the identity matrix, I. Note, however, that the methods considered in this paper will not be affected by this model assumption because we are solving the regression problem using a least squares approach. This entails that the obtained regression models do not depend on the model parameter $\boldsymbol{\Sigma}_{\boldsymbol{\epsilon}_{x \mid d}}[12]$.

\section{Ensemble Kalman filtering using kernel-shrinkage regression}

We will now present the kernelised versions of the EnKF updating scheme based on three common shrinkage regression techniques; ridge regression (RR) [14], PCR and PLSR. However, we will only consider kernel $\mathrm{RR}$ in detail. This is motivated because kernel RR is connected to the probabilistic solution of the nonlinear regression problem discussed above; details are given below. The interested reader can find a thorough description of the PCR and PLSR shrinkage regression techniques in Sætrom and Omre [46].

For simplicity, we define kernel matrices $\boldsymbol{C}^{*}=$ $\left[\boldsymbol{c}^{*}, \ldots, \boldsymbol{c}^{*}\right] \in \mathbb{R}^{n_{e} \times n_{e}}$, with $\boldsymbol{c}^{*}=\left[c\left(\boldsymbol{d}^{(1)}, \boldsymbol{d}^{*}\right), \ldots, c\left(\boldsymbol{d}^{\left(n_{e}\right)}\right.\right.$, $\left.\left.\boldsymbol{d}^{*}\right)\right]^{T} \in \mathbb{R}^{n_{e} \times 1}$, and

$\boldsymbol{C}=\left[\begin{array}{ccc}c\left(\boldsymbol{d}^{(1)}, \boldsymbol{d}^{(1)}\right) & \ldots & c\left(\boldsymbol{d}^{(1)}, \boldsymbol{d}^{\left(n_{e}\right)}\right) \\ \vdots & \ddots & \vdots \\ c\left(\boldsymbol{d}^{\left(n_{e}\right)}, \boldsymbol{d}^{(1)}\right) & \ldots & c\left(\boldsymbol{d}^{\left(n_{e}\right)}, \boldsymbol{d}^{\left(n_{e}\right)}\right)\end{array}\right]$.

For all three methods, we find the estimated Kalman gain matrix by solving the following multivariate linear regression problem in a feature space, $\mathcal{F}$ :

$\boldsymbol{x}=\boldsymbol{K} \varphi(d)+\boldsymbol{\epsilon}_{\boldsymbol{x} \mid \boldsymbol{d}}$,

where $\boldsymbol{\epsilon}_{\boldsymbol{x} \mid \boldsymbol{d}} \in \mathbb{R}^{n_{x} \times 1}$ represents the regression model error. Similar to above, $\boldsymbol{\Phi}=\left[\boldsymbol{\varphi}\left(\boldsymbol{d}^{(1)}\right), \ldots, \boldsymbol{\varphi}\left(\boldsymbol{d}^{\left(n_{e}\right)}\right)\right]$, and we assume that all vectors and ensemble matrices are centred, unless otherwise stated.

\subsection{Kernel ridge regression}

Kernel RR is a regularisation method where we select the estimated regression coefficients by minimising the mean squared error with additional constraints, that is:

$$
\begin{gathered}
\hat{\boldsymbol{K}}_{\mathrm{KerRR}}=\arg \min _{\boldsymbol{K}}\left\{\operatorname{tr}\left\{(\boldsymbol{X}-\boldsymbol{K} \boldsymbol{\Phi})(\boldsymbol{X}-\boldsymbol{K} \boldsymbol{\Phi})^{T}\right\}\right. \\
\left.+\xi \operatorname{tr}\left\{\boldsymbol{K} \boldsymbol{K}^{T}\right\}\right\} .
\end{gathered}
$$

Solving this minimisation problem analytically gives [42]:

$$
\begin{aligned}
\hat{\boldsymbol{K}}_{\mathrm{KerRR}} & =\boldsymbol{X} \boldsymbol{\Phi}^{T}\left(\boldsymbol{\Phi} \boldsymbol{\Phi}^{T}+\xi \boldsymbol{I}\right)^{-1} \\
& =\boldsymbol{X}\left(\boldsymbol{\Phi}^{T} \boldsymbol{\Phi}+\xi \boldsymbol{I}\right)^{-1} \boldsymbol{\Phi}^{T} .
\end{aligned}
$$

Thus, we obtain kernel RR predictions for an unknown $\boldsymbol{x}^{*}$, with associated $\boldsymbol{d}^{*}$, based on

$\boldsymbol{x}^{*}=\boldsymbol{X}(\boldsymbol{C}+\xi \boldsymbol{I})^{-1} \boldsymbol{c}^{*}$

Comparing this expression with Eq. 13, we see that the two predictors, $\boldsymbol{x}^{*}$ and $E\left[\boldsymbol{x}^{*} \mid \boldsymbol{X}\right]$ are identical for $\sigma_{x \mid \boldsymbol{d}}^{2}=\xi$. This follows because each variable $\boldsymbol{x}_{i}^{*}$, for $i=1, \ldots, n_{x}$, can be computed independently. Thus, applying kernel RR in an EnKF setting leads to the following updating scheme:

$\boldsymbol{X}^{c}=\boldsymbol{X}^{u}+\boldsymbol{X}^{u} \boldsymbol{H}(\boldsymbol{H C H}+\xi \boldsymbol{I})^{-1} \boldsymbol{H}\left(\boldsymbol{C}^{*}-\boldsymbol{C}\right)$.

Here, $\boldsymbol{X}^{c}$ and $\boldsymbol{X}^{u}$ are the state ensemble matrices, conditioned and unconditioned respectively and $\boldsymbol{H} \boldsymbol{C H}$ is the centred kernel matrix [41], with the centring matrix, $\boldsymbol{H}$, defined in Eq. 6.

\subsection{Kernel principal component regression}

PCR is based on the assumption that most of the variability in the predictor variables can be explained through a small set of random variables, termed principal components. The estimated matrix of regression coefficients is then constructed using the principal components as predictor variables. Hence, we define a matrix of regression coefficients in a reduced order space. Kernel PCR follows directly by applying kernel PCA $[35,41]$ to $\varphi(d)$. The resulting expression for the estimated Kalman gain matrix is

$\hat{\boldsymbol{K}}_{\mathrm{KerPCR}}=\boldsymbol{X} \boldsymbol{E}_{p} \boldsymbol{\Lambda}_{p}^{-1} \boldsymbol{E}_{p}^{T} \boldsymbol{\Phi}^{T}$.

Here $\boldsymbol{E}_{p} \in \mathbb{R}^{n_{e} \times p}$ contains the $p$ eigenvectors of the centred kernel matrix, $\boldsymbol{H C H}$, with the $p$ largest corresponding eigenvalues given in the diagonal matrix, $\boldsymbol{\Lambda}_{p} \in \mathbb{R}^{p \times p}$. The following expression then forms an EnKF updating scheme based on kernel PCR:

$$
\boldsymbol{X}^{c}=\boldsymbol{X}^{u}+\boldsymbol{X}^{u} \boldsymbol{E}_{p} \boldsymbol{\Lambda}_{p}^{-1} \boldsymbol{E}_{p}^{T}\left(\boldsymbol{C}^{*}-\boldsymbol{C}\right) .
$$

\subsection{Kernel partial least squares regression}

Similar to PCR, PLSR applies dimension reduction techniques to the predictor variables. The main difference between the two methods is that, PLSR uses the information available from both the response 
and predictor variables when projecting into the $p$ dimensional subspace, which entails that PLSR is based on a supervised dimension reduction technique. The kernelised version of the PLSR algorithm follows from the algorithm presented in Ränner et al. [34]. This results in the following estimate of the Kalman gain matrix:

$\hat{\boldsymbol{K}}_{\mathrm{KerPLSR}}=\boldsymbol{X} \boldsymbol{T} \boldsymbol{A}^{-1} \boldsymbol{W}^{T} \boldsymbol{\Phi}^{T}$.

Here, $\boldsymbol{A}=\left(\boldsymbol{W}^{T} \boldsymbol{C T}\right) \in \mathbb{R}^{p \times p}$, with latent variables $\boldsymbol{T}=\left[\boldsymbol{t}_{1}, \ldots, \boldsymbol{t}_{p}\right] \in \mathbb{R}^{n_{e} \times p}$ and $\boldsymbol{W}=\left[\boldsymbol{w}_{1}, \ldots, \boldsymbol{w}_{p}\right] \in \mathbb{R}^{n_{e} \times p}$ given by solving sequentially for $i=1, \ldots, p$ :

$\left[\begin{array}{c}\boldsymbol{t}_{i}=\boldsymbol{H} \boldsymbol{\Phi}^{T} \boldsymbol{\psi}_{i} \\ \boldsymbol{w}_{i}=\boldsymbol{H} \boldsymbol{X}^{T} \boldsymbol{v}_{i}\end{array}\right] \leftarrow\left\{\begin{array}{l}\max _{\boldsymbol{\psi}_{i}, \boldsymbol{v}_{i}}\left\{\boldsymbol{v}_{i}^{T} \boldsymbol{X} \boldsymbol{H} \boldsymbol{\Phi}^{T} \boldsymbol{\psi}_{i}\right\} \\ \left\|\boldsymbol{\psi}_{i}\right\|_{2}=1,\left\|\boldsymbol{v}_{i}\right\|_{2}=1 \\ \boldsymbol{t}_{i}^{T} \boldsymbol{t}_{j}=0, \text { for all } j<i\end{array}\right.$

Ränner et al. [34] outline an efficient procedure for solving this problem when $n_{x}$ and $n_{d}$ are larger than $n_{e}$. This gives the following EnKF updating scheme based on kernel PLSR:

$\boldsymbol{X}^{c}=\boldsymbol{X}^{u}+\boldsymbol{X}^{u} \boldsymbol{T} \boldsymbol{A}^{-1} \boldsymbol{W}^{T}\left(\boldsymbol{C}^{*}-\boldsymbol{C}\right)$,

with $\boldsymbol{A}$ defined above. Note that centring of the ensemble matrices is unnecessary because of the identities $\boldsymbol{T}=\boldsymbol{H} \boldsymbol{T}$ and $\boldsymbol{W}=\boldsymbol{H} \boldsymbol{W}[46]$.

\subsection{Comments}

Although the three shrinkage regression techniques presented above, and their kernelised versions, all reduce the problems caused by collinearities in the data ensemble, they accomplish this differently. Note that collinearities in the data ensemble will result in small eigenvalues in the estimated data covariance matrix, $\hat{\boldsymbol{\Sigma}}_{d}$, or the corresponding centred kernel matrix, $\boldsymbol{H C H}$, which can result in large weights in the respective Kalman gain matrices. Whilst the RR technique reduces the weight caused by smallest eigenvalues by adding a positive constant to all eigenvalues, the PCR technique eliminates small eigenvalues, whilst retaining the $p$ dominant. The PLSR technique works in a similar manner as the PCR, although the analysis is slightly more complicated (see Hastie et al. [12] for a detailed description). However, it has been noted that because the PLSR technique uses the information from both the state and data ensemble matrices in the dimension reduction, a small number of components, $p$, is often required in PLSR compared with PCR $[13,20]$.

It should be noted that the optimality of the different shrinkage regression techniques appears to be problem dependent. That is, whilst RR leads to the smallest prediction error in one study, PCR or PLSR can be optimal in other studies. It is therefore advisable to test the predictive performance of the different methods based on the prior and likelihood model, before selecting which method to use in an EnKF setting. Finally it is important to note that the performance of all three schemes are highly dependent on the model hyperparameters used. Thus, schemes which enables an automatic selection of these model parameters are called for.

\subsection{Model hyperparameter selection}

The results obtained using the kernel-shrinkage regression techniques described above, will in general depend on one or more hyperparameters, $\boldsymbol{\theta}$. For kernel RR this involves selecting the size of the regularisation parameter, $\xi$, whilst for kernel PCR and PLSR the dimension of the reduced order space, $p$, is required. In addition, we need to select the kernel function, $c(\cdot, \cdot)$, which implicitly defines $\varphi(\cdot)$. This can be challenging if all symmetric, positive definite kernel functions are considered.

The usual approach for selecting $c(\cdot, \cdot)$ is to consider only certain families of kernel functions. An example is translation and rotation invariant functions $c\left(\boldsymbol{d}, \boldsymbol{d}^{\prime}\right)=$ $c\left(\left\|\boldsymbol{d}-\boldsymbol{d}^{\prime}\right\|_{2}^{2}\right)$, referred to as radial basis function (RBF) kernels, where $\|\cdot\|_{2}$ is the Euclidean norm [44]. The second order exponential kernel function, defined in Eq. 11, is an example of such a kernel function. Another example of a kernel function is the polynomial, defined as $c\left(\boldsymbol{d}, \boldsymbol{d}^{\prime}\right)=\left(1+\boldsymbol{d}^{T} \boldsymbol{d}^{\prime}\right)^{v}, v>1$, which entails that $\varphi(\cdot)$ represents polynomials. Common for most of the kernel functions in the literature, however, is that we only need to specify one or two hyperparameters.

To avoid overfitting the regression model to the data, we can apply cross-validation (CV), as discussed in Sætrom and Omre [46]. CV works by sequentially splitting the state vector and data ensembles into training ensemble matrices, $\left(\boldsymbol{X}_{\text {Train }}, \boldsymbol{D}_{\text {Train }}\right)$, used for model estimation, and test ensembles matrices, ( $\left.\boldsymbol{X}_{\text {Test }}, \boldsymbol{D}_{\text {Test }}\right)$, used for model validation. Using the training ensemble matrices to estimate the Kalman gain matrix for model parameters $\boldsymbol{\theta}, \hat{\boldsymbol{K}}_{\text {Train }}(\boldsymbol{\theta})$, we can predict the state vectors based on the test data, $\hat{\boldsymbol{X}}_{\text {Test }}=\hat{\boldsymbol{K}}_{\text {Train }}(\boldsymbol{\theta}) \boldsymbol{D}_{\text {Test }}$. Computing the sum of squares of the mismatch between $\hat{\boldsymbol{X}}_{\text {Test }}$ and $\boldsymbol{X}_{\text {Test }}$, referred to as the predictive error sum of squares (PRESS) statistic, can be used to measure of the predictive power for the chosen model. Note, however, that it is straightforward to use other objective functions than the sum of squares. We can then 
select the model hyperparameters by minimising the PRESS statistic for different values of $\boldsymbol{\theta}$, as discussed below. A pseudo-code describing the workflow of the EnKF updating scheme based on kernel PCR, using $m$-fold CV to select the model parameters is given in Algorithm 1. Note that we here use the notation $\boldsymbol{I}_{i}$ to denote the set of indices for members of test ensemble $i$. A similar workflow can be used for the RR and PLSR techniques as well.

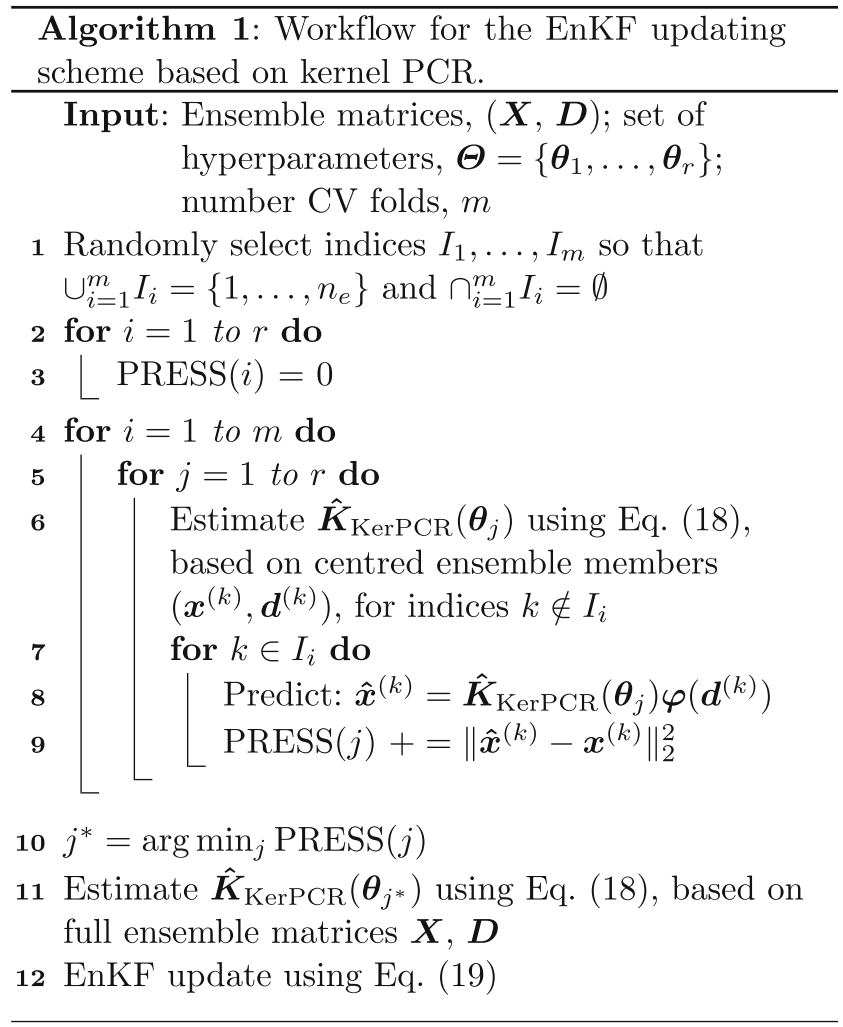

The model parameters, $\boldsymbol{\theta}$, will for most applications of kernel-shrinkage regression contain two parameters, which can be both discrete and continuous. Alternative methods for solving this problem are gradient based optimisation techniques [29], or stochastic optimisation techniques such as particle swarm optimisation [22] and simulated annealing [23]. In practise, however, it is often sufficient to consider a limited number discrete values for the continuous model parameters, for example $\Delta \in\{1,2, \ldots, 20\}$ for the second order exponential covariance function, rather than searching for the global optimum [44]. Hence, we do not necessarily suffer from high computational demands if we select the model parameters based on an exhaustive search rather than sophisticated optimising schemes. Also note that for applications of the EnKF where it is extremely time consuming to evaluate the forward model $\omega(\cdot)$, such as petroleum reservoir characterisation, the use of $\mathrm{CV}$ for model parameter selection will not increase the total computational time significantly. In addition, we emphasise that the $\mathrm{CV}$ scheme is straightforward to run in parallel. A discussion regarding the computational properties of the EnKF updating scheme based on kernel-shrinkage regression techniques can be found in Appendix B.

Finally, because the likelihood model is known, selection of the kernel function is easier in this setting, compared with non-linear regression problems where the relation between $\boldsymbol{d}$ and $\boldsymbol{x}$ is unknown. An alternative approach is therefore to select the kernel function based on prior knowledge of the state vector and the likelihood model. That is, we select the hyperparameters of the kernel function based on the predictive power of the non-linear regression model created using realisations from the initial prior model.

\section{Empirical study}

We define the state vector $\boldsymbol{x}_{k} \in \mathbb{R}^{100 \times 1}, k=0, \ldots, 19$ on a $(10 \times 10)$ regular grid domain. Here, $x_{i, j, k}$ denotes the value of the state vector at location $(i, j), i=1, \ldots, 10$, $j=1, \ldots, 10$ and time step $k$. We assume that the state vector is static, meaning $\boldsymbol{x}_{k+1}=\boldsymbol{x}_{k}, k=0, \ldots, 19$, with a reference model generated from the Gaussian prior model:

$\boldsymbol{x}^{\text {True }} \sim \operatorname{Gauss}_{n_{x}}\left(\boldsymbol{\mu}_{\boldsymbol{x}}, \boldsymbol{\Sigma}_{\boldsymbol{x}}\right)$.

Here, $\mu_{x}=5 \times \mathbf{1}$, and we construct $\boldsymbol{\Sigma}_{x}$ based on an exponential covariance function,

$\operatorname{Cov}\left(x_{i, j, 0}, x_{l, m, 0}\right)=\exp \left\{-3(\boldsymbol{\Delta})^{1.2}\right\}$,

where

$\boldsymbol{\Delta}=\sqrt{\left(\frac{\Delta_{x}}{l_{x}}\right)^{2}+\left(\frac{\Delta_{y}}{l_{y}}\right)^{2}}$.

The range parameters $l_{x}$ and $l_{y}$ are one and 10 , respectively.

We use the non-linear likelihood model:

$\boldsymbol{d}_{k}=\zeta\left(\boldsymbol{x}, \boldsymbol{\epsilon}_{\boldsymbol{d}}\right)$,

to connect the observations $\boldsymbol{d}_{k}$ to the state vector. Here each element $\zeta_{i, j, k}=g\left(x_{i, j, k}, 5\right)+\sigma_{d} \epsilon_{i, j, k}^{2}$, with $g(\cdot)$ defined as:

$g(x, \beta)=\left\{\begin{array}{cc}\frac{\sin (|x-\beta|) \cos (d)}{|x-\beta|}, & x \neq \beta \\ 1, & x=\beta .\end{array}\right.$,

and $\epsilon_{i, j, k} \sim \operatorname{Gauss}_{1}(0,1)$. Hence, we have an additive error term $\epsilon_{d} / \sigma_{d} \sim \chi_{1}^{2}$, where $\chi_{v}^{2}$ denotes the 
$\chi^{2}$-distribution with $v$-degrees of freedom [4], which makes the likelihood function both non-Gaussian and non-linear. Here $\sigma_{d}=0.25$, which implies that the mean and variance of the error term are equal to 0.250 and 0.125 , respectively. Figure 3 shows an image plot of the reference state vector. The scatter plot of $\boldsymbol{x}^{\text {True }}$ and the corresponding observed data at the initial time step, $\boldsymbol{d}_{0}$, in Fig. 4, displays the non-linear structure of the likelihood model. At each time step $k=0, \ldots, 19$, we make new observations, $\boldsymbol{d}_{k}$.

\subsection{Non-linear regression}

To demonstrate the effect of the suggested kernel regression techniques we initially consider a univariate non-linear regression problem, with a bivariate predictor variable, based on the function defined in Eq. 23 as follows: Define the date ensemble matrix, $\boldsymbol{D}$, through $n_{e}=91$ uniformly spaced points, $\boldsymbol{d} \in \mathbb{R}^{2 \times 1}$, with $d_{1}$ and $d_{2}$ having values between -4 and 4 . Assume that at each of these 91 locations, we observe values of the function,

$x=\gamma(\boldsymbol{d})+\epsilon_{x \mid \boldsymbol{d}}=1+g\left(d_{1}, 0\right)+g\left(d_{2}, 0\right)+\epsilon_{x \mid \boldsymbol{d}}$,

where the noise term, $\epsilon_{x \mid \boldsymbol{d}}$, is Gaussian having zero mean and standard deviation 0.5. Figures 5 and 6 display the reference solution and the observed data.

Using the ensemble matrices $\boldsymbol{D}$ and $\boldsymbol{X}$, the task is to fit a regression model based on the classical linear least squares approach and the three kernel-shrinkage regression models discussed above. Because the Gaussian kernel function, described in Eq. 14, is a robust choice when no prior information is available regarding the

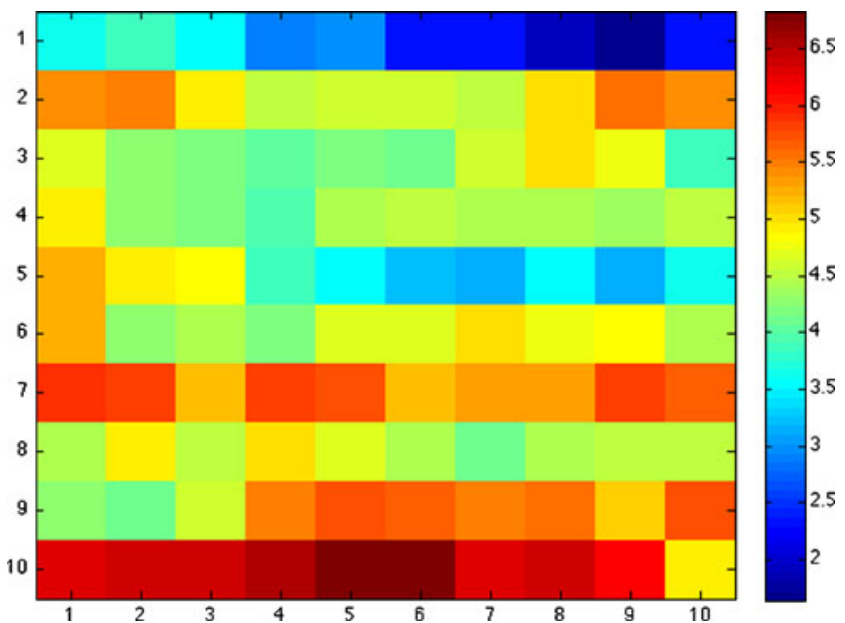

Fig. 3 Reference state vector used in the empirical case study generated from a Gaussian prior distribution with an anisotropic covariance function

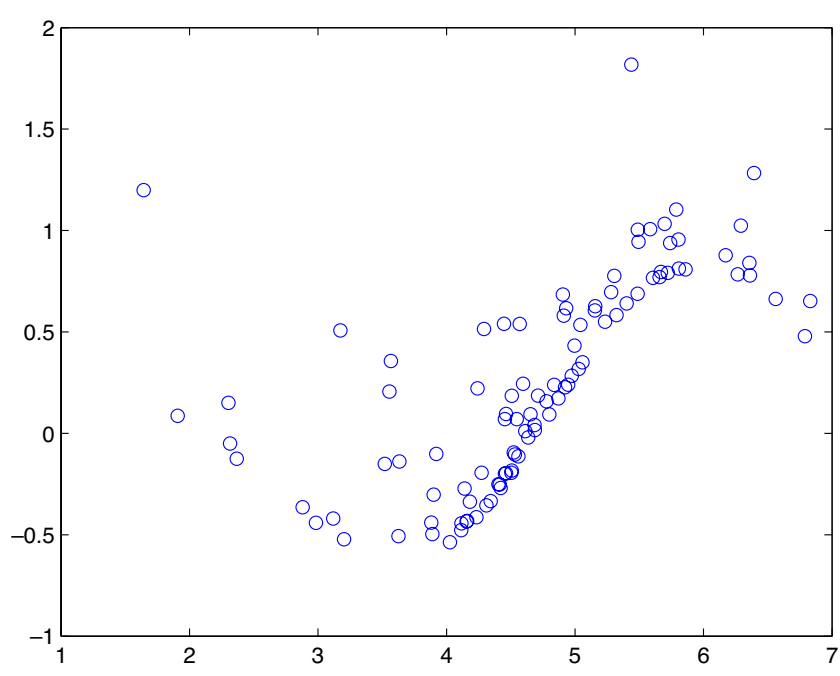

Fig. 4 Scatter plot between the elements of $\boldsymbol{x}^{\text {True }}$ and corresponding observations, $\boldsymbol{d}^{o}$, based on the non-linear, nonGaussian likelihood model

data [43, 45], we use it to map $\boldsymbol{d}$ into the feature space $\mathcal{F}$. To select the scaling factor in the kernel function and shrinkage factor for the three regression methods, we apply 10 -fold CV. Figure 7 display the results. Note that because the prior and likelihood models are fully specified, another alternative is to tailor the kernel function based on this known prior information. How this task can be accomplished in an automatic manner is, however, somewhat unclear and is a topic for further research. Hence, we apply the Gaussian kernel function for the case studies considered in this paper, even if this selection might be sub-optimal.

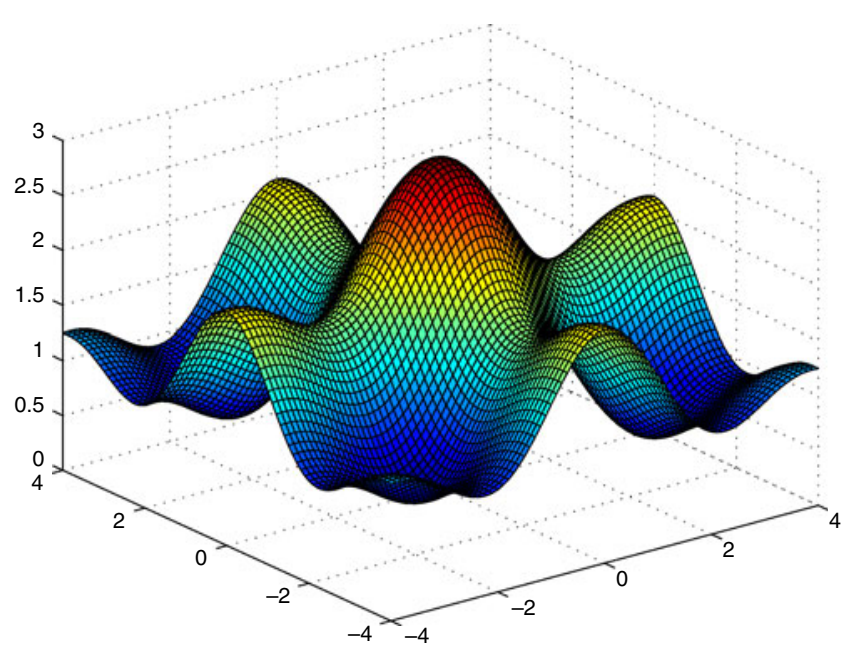

Fig. 5 Reference solution for the non-linear regression problem considered, $x=\gamma(\boldsymbol{d})$, for locations, $\boldsymbol{d}$ in the plane 


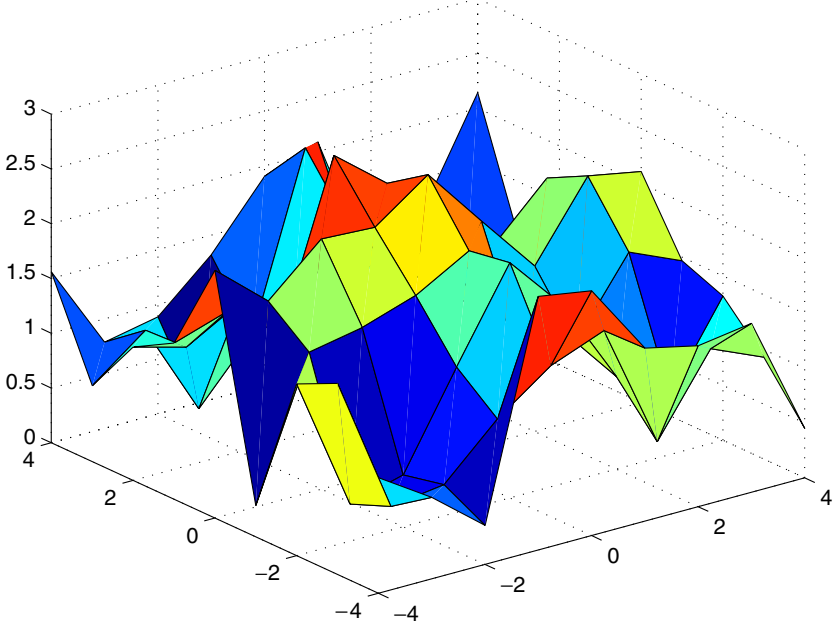

Fig. 6 Observed data of the function $x=\gamma(\boldsymbol{d})+\epsilon_{x \mid \boldsymbol{d}}$ at 81 uniformly spaced locations $\boldsymbol{d}$ in the plane

As expected, the plane resulting from the linear least squares method, does not provide a good prediction of the non-linear function. The three kernel methods, on the other hand, are able to capture the non-linear trend of the function, thus providing reasonable predictions. Figure 8 display the absolute deviance between the reference and predicted solution for the four methods. Except for the larger error occurring at the origin for the kernel RR method, the deviance is within one standard deviation of the observation error at all predicted
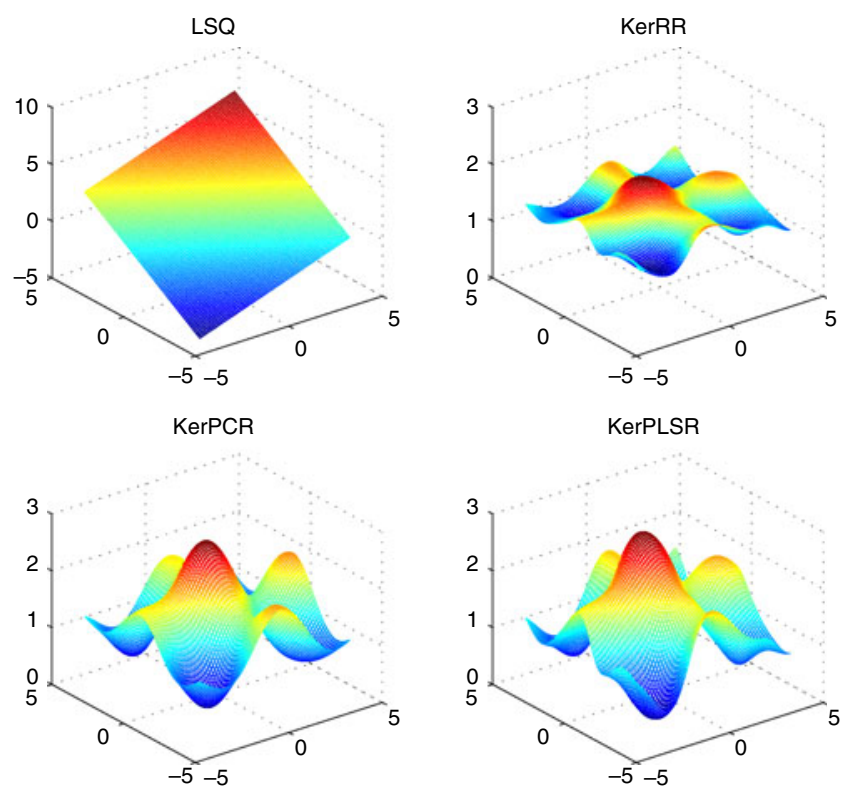

Fig. 7 Predicted outcome based on least squares linear regression $(L S Q)$, kernel RR (KerRR), kernel PCR (KerPCR) and kernel PLSR (KerPLSR); 10-fold CV was used to select the prior hyperparameters $\boldsymbol{\theta}$
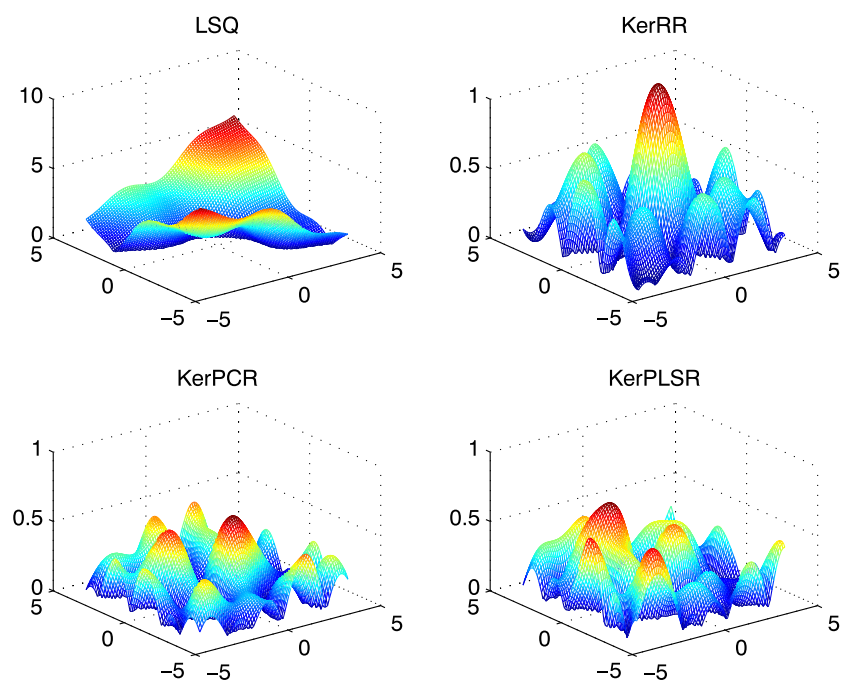

Fig. 8 Absolute deviance between the true solution and the predicted outcome based on least squares linear regression $(L S Q)$, kernel RR (KerRR), kernel PCR (KerPCR) and kernel PLSR (KerPLSR). 10-fold CV was used to select the prior hyperparameters $\boldsymbol{\theta}$

locations using the three kernel methods. The main difference between the PCR and PLSR approaches is, however, that the former requires $p=13$ components, whilst the latter only requires $p=1$. Note that this behaviour is in accordance with the discussion above, because PLSR uses information from both the stateand data vector in the dimension reduction.

\subsection{Results}

Based on the results seen in the non-linear regression based on the likelihood model, we proceed using the EnKF based on kernel PCR and PLSR techniques on the filtering problem with the Gaussian kernel function defined in Eq. 14. We use the following sampling scheme to generate the initial ensemble with $n_{e}=100$ :

$$
\begin{aligned}
& \boldsymbol{x}^{u(1)}=10 \times \mathbf{1} \\
& \boldsymbol{x}^{u(2)}, \ldots, \boldsymbol{x}^{u(25)} \sim \operatorname{Gauss}_{n_{x}}\left(\frac{1}{1.5} \boldsymbol{\mu}_{\boldsymbol{x}}, 2 \boldsymbol{\Sigma}_{\boldsymbol{x}}\right) \\
& \boldsymbol{x}^{u(26)}, \ldots, \boldsymbol{x}^{u(50)} \sim \operatorname{Gauss}_{n_{x}}\left(\frac{1}{2} \boldsymbol{\mu}_{\boldsymbol{x}}, 2 \boldsymbol{\Sigma}_{\boldsymbol{x}}\right) \\
& \boldsymbol{x}^{u(51)}, \ldots, \boldsymbol{x}^{u(75)} \sim \operatorname{Gauss}_{n_{x}}\left(2 \boldsymbol{\mu}_{\boldsymbol{x}}, 2 \boldsymbol{\Sigma}_{\boldsymbol{x}}\right) \\
& \boldsymbol{x}^{u(76)}, \ldots, \boldsymbol{x}^{u(100)} \sim \operatorname{Gauss}_{n_{x}}\left(1.5 \boldsymbol{\mu}_{\boldsymbol{x}}, 2 \boldsymbol{\Sigma}_{\boldsymbol{x}}\right),
\end{aligned}
$$

with $\boldsymbol{\mu}_{\boldsymbol{x}}$ and $\boldsymbol{\Sigma}_{\boldsymbol{x}}$ defined above. Hence, there is a high uncertainty regarding the mean of the true underlying distribution. The purpose of this study is to assimi- 
late observed data using the standard EnKF updating scheme for non-linear observations [8, Appendix A.2] and the EnKF updating schemes based on kernel-shrinkage regression. Because $n_{d}=n_{e}, \hat{\Sigma}_{d}$ will be singular. We therefore add a positive definite regularisation term before inverting the matrix, as explained in Section 3, with $\boldsymbol{\Sigma}_{\boldsymbol{r}}=2 \sigma_{d}^{2} \boldsymbol{I}$.

When selecting prior hyperparameters in the kernelshrinkage regression techniques, $\boldsymbol{\theta}_{k}$, we consider the following two approaches:

- Automatic: $\boldsymbol{\theta}=\left(\sigma^{2}, p\right)$ selected based on 10-fold $\mathrm{CV}$ at each time step, minimising the PRESS statistic.

- Supervised: $\boldsymbol{\theta}=\left(\sigma^{2}, p\right)$ selected based is based on 10 -fold $\mathrm{CV}$ at the initial time step, minimising the PRESS statistic, and remain fixed for all time steps $k=0, \ldots, 19$.
Hence, we consider five different EnKF updating schemes in total:

- Classical EnKF: The non-linear formulation of the EnKF updating scheme using the estimated Kalman gain matrix given in Eq. 9, assuming a Guassian additive noise term: $\boldsymbol{d}=\boldsymbol{\zeta}(\boldsymbol{x})+\boldsymbol{\epsilon}_{\boldsymbol{d}}$, where $\boldsymbol{\epsilon}_{\boldsymbol{d}} \sim \operatorname{Gauss}_{n_{d}}\left(\mathbf{0}, 2 \sigma_{d}^{2} \boldsymbol{I}\right)$. This implies that the covariance of the noise term, $\boldsymbol{\Sigma}_{r}$, is equal to the true variance of the likelihood model [4], although we do not account for the non-zero mean.

- EnKF-KerPLSR1: EnKF based on the kernelised PLSR method. The dimension of the reduced order space, and the scaling parameter in the Gaussian kernel function selected based on the automatic approach

- EnKF-KerPCR1: EnKF based on the kernelised PCR method. The dimension of the reduced order

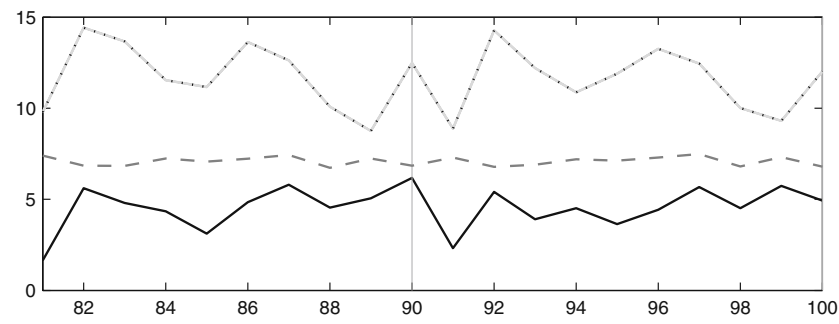

(a) Classical EnKF

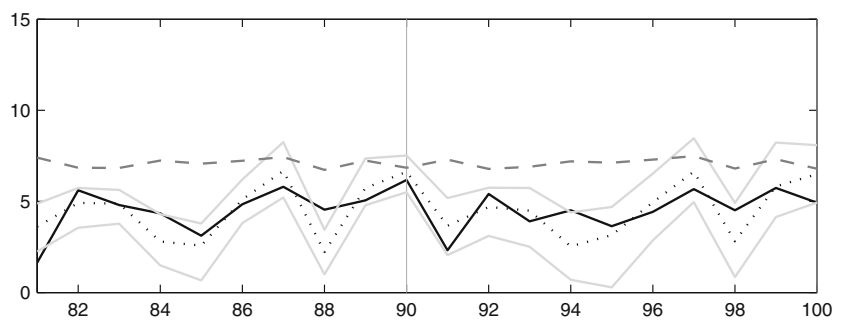

(b) EnKF-KerPLSR1

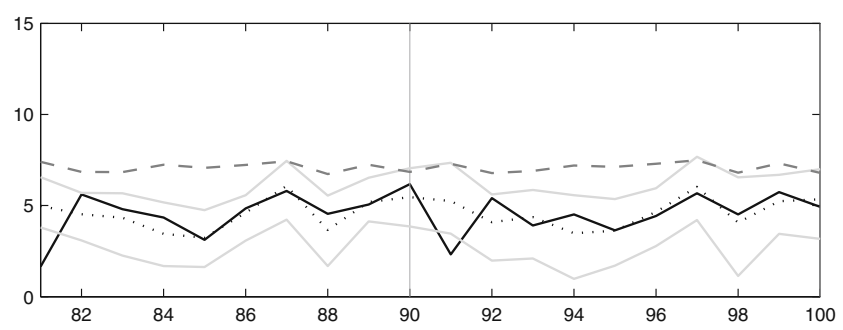

(d) EnKF-KerPLSR2

Fig. 9 Results obtained when running five different EnKF updating schemes on a problem with a highly non-linear likelihood model. The figure displays, for grid nodes 81 through 100, the reference $\boldsymbol{x}_{10}^{\text {True }}$ (solid), the ensemble average (dotted) and the es-

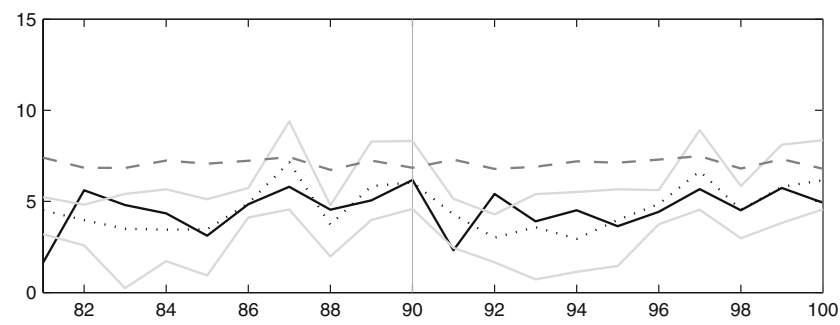

(c) EnKF-KerPCR1

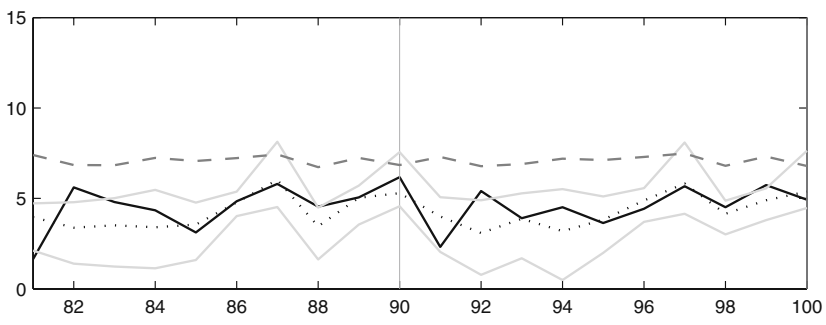

(e) EnKF-KerPCR2

timated $95 \%$ confidence bounds of the prediction interval (solid, light grey) at the final time step. The ensemble mean at the initial time step is shown as the dashed, dark grey line 
space, and the scaling parameter in the Gaussian kernel function selected based on the automatic approach

- EnKF-KerPLSR2: EnKF based on the kernelised PLSR method. The dimension of the reduced order space, and the scaling parameter in the Gaussian kernel function selected based on the supervised approach.

- EnKF-KerPCR2: EnKF based on the kernelised PCR method. The dimension of the reduced order space, and the scaling parameter in the Gaussian kernel function selected based on the supervised approach

Figure 9a-e, displays the results obtained in grid nodes 81 through 100 using the five different EnKF updating schemes. The results are similar for the remaining locations, and are therefore not included. Note that the initial ensembles are identical for all five schemes.

As we can see from Fig. 9a, the classical EnKF is not able to get a good representation of the state vector at the final updating step. The estimated posterior mean is farther from the reference state vector than at the initial time step and the ensemble has collapsed into a single realisation. Because we are using a linear updating scheme on a non-linear likelihood model, we expect that the ensemble mean is missing the reference state vector. However, it is more troubling that we are not able to obtain estimates of the prediction uncertainty. Ideally, the estimated prediction interval based on the updated ensemble, should cover $95 \%$ of the reference state vector. In this case, however, the coverage is $0 \%$.

The EnKF-KerPLSR1 method is able to obtain reasonable estimates of the reference state vector, $\boldsymbol{x}^{\text {True }}$. The estimated posterior mean is centred around $\boldsymbol{x}^{\text {True }}$, and the estimated prediction interval is giving a good description of the solution uncertainty. The EnKFKerPCR1, EnKF-KerPLSR2 and EnKF-KerPCR2 updating schemes have a similar behaviour, as shown in Fig. 9c-e.

Figure 10 contains two realisations of the state vector in the initial ensemble and the corresponding re-
Fig. 10 Two realisations and the estimated ensemble mean for the initial ensemble, the classical EnKF updating scheme $(C$-EnKF) and the four kernel-shrinkage regression techniques
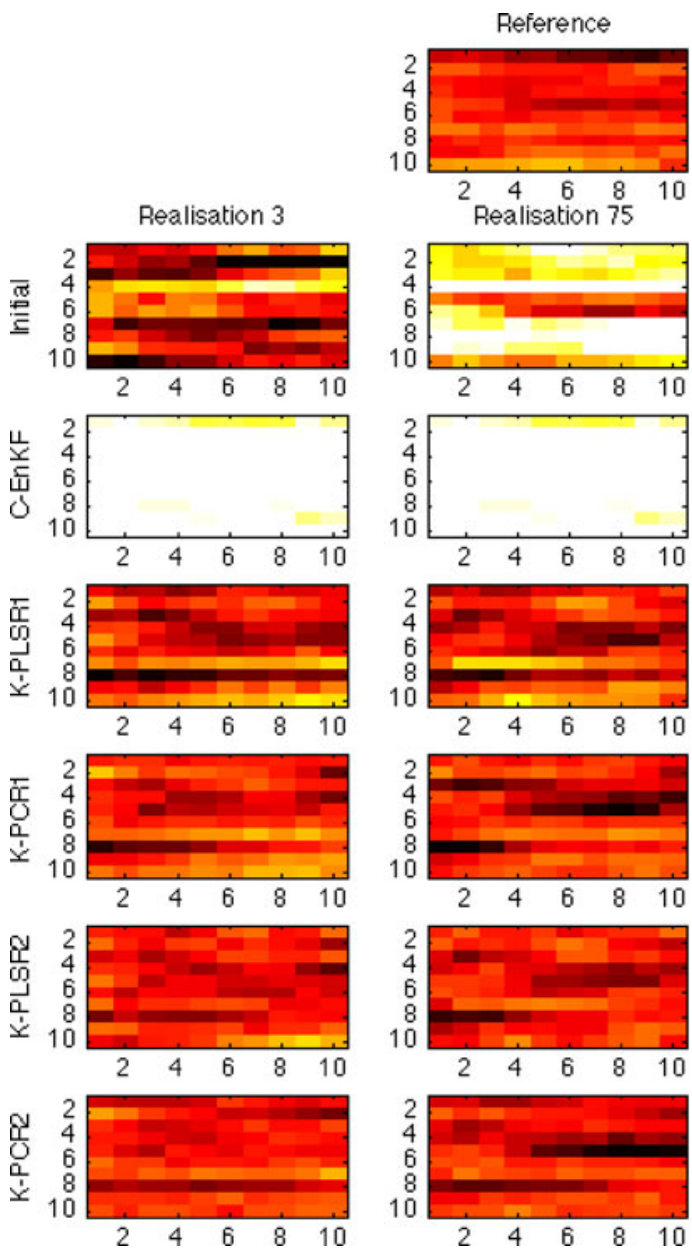
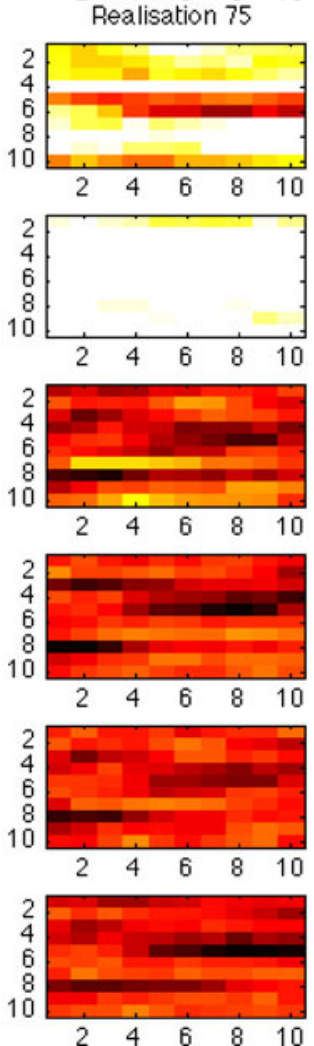
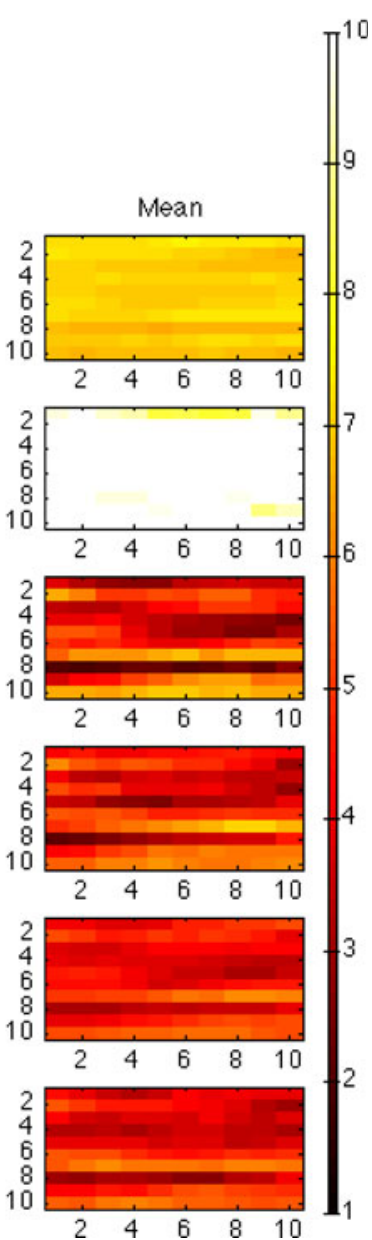
Table 1 Scaled $\left(1 / n_{e}\right)$ total sum of squares (TSS) of the estimated posterior mean to the reference solution, and coverage of the reference solution in the estimated $95 \%$ prediction intervals based on 100 different initial ensembles

\begin{tabular}{llrc}
\hline$n_{e}$ & Scheme & TSS & $\begin{array}{l}\text { Coverage } \\
(\%)\end{array}$ \\
\hline 100 & No updating & 70.6 & 100.0 \\
100 & Classical EnKF & 311.5 & 6.1 \\
100 & EnKF-KerPLSR1 & 9.3 & 92.2 \\
100 & EnKF-KerPCR1 & 9.0 & 96.6 \\
100 & EnKF-KerPLSR2 & 7.5 & 93.6 \\
100 & EnKF-KerPCR2 & 6.2 & 95.6 \\
100 & EnKF-PLSR1 & 60.5 & 19.3 \\
\hline
\end{tabular}

alisations after the final updating step, using the five different EnKF updating schemes. We also display the ensemble mean. At the initial time step, the realisations and the ensemble mean do not resemble the reference state vector, as expected from the prior distribution described above. Again, we notice that the realisations obtained using the classical EnKF scheme has collapsed, and are farther from $\boldsymbol{x}^{\text {True }}$, than they were initially. The four kernel-shrinkage regression techniques, on the other hand, appear to give a good representation of the reference state vector. We especially note that the anisotropic behaviour in $\boldsymbol{x}^{\text {True }}$ is captured in both the realisations and the ensemble mean for all of the four schemes based on kernel-shrinkage regression.

It is interesting to note that the results obtained using the automatic and supervised methods to select $\theta$ produces similar results. For the supervised parameter selection approach $\boldsymbol{\theta}$ was selected as: $\theta_{\mathrm{PCR}}=(10,5)$ and $\boldsymbol{\theta}_{\mathrm{PLSR}}=(10,1)$, whilst for the $\mathrm{CV}$ based selection scheme $\boldsymbol{\theta}_{\mathrm{PCR}} \in\{[1,7](5),[5,30](20)\}$ and $\boldsymbol{\theta}_{\mathrm{PLSR}} \in$ $\{[1,5](1),[5,30](20)\}$. Here the notation $[1,5](5)$ is used to denote the smallest, largest and median value of the selected parameters respectively.

To quantify the performance of the five updating schemes the model is rerun 100 times using different initial ensembles. We then compute the scaled total sum of squares (TSS), given as $1 / n_{e} \sum_{i=1}^{n_{e}} \| \hat{\boldsymbol{\mu}}_{\boldsymbol{x}^{c}}-$ $\boldsymbol{x}^{\text {True }} \|_{2}^{2}$, and the coverage of the reference solution within the estimated $95 \%$ prediction intervals. The results are summarised in Table 1 . Here we have included the results obtained using PLSR regression in the Euclidean space, rather than the feature space,

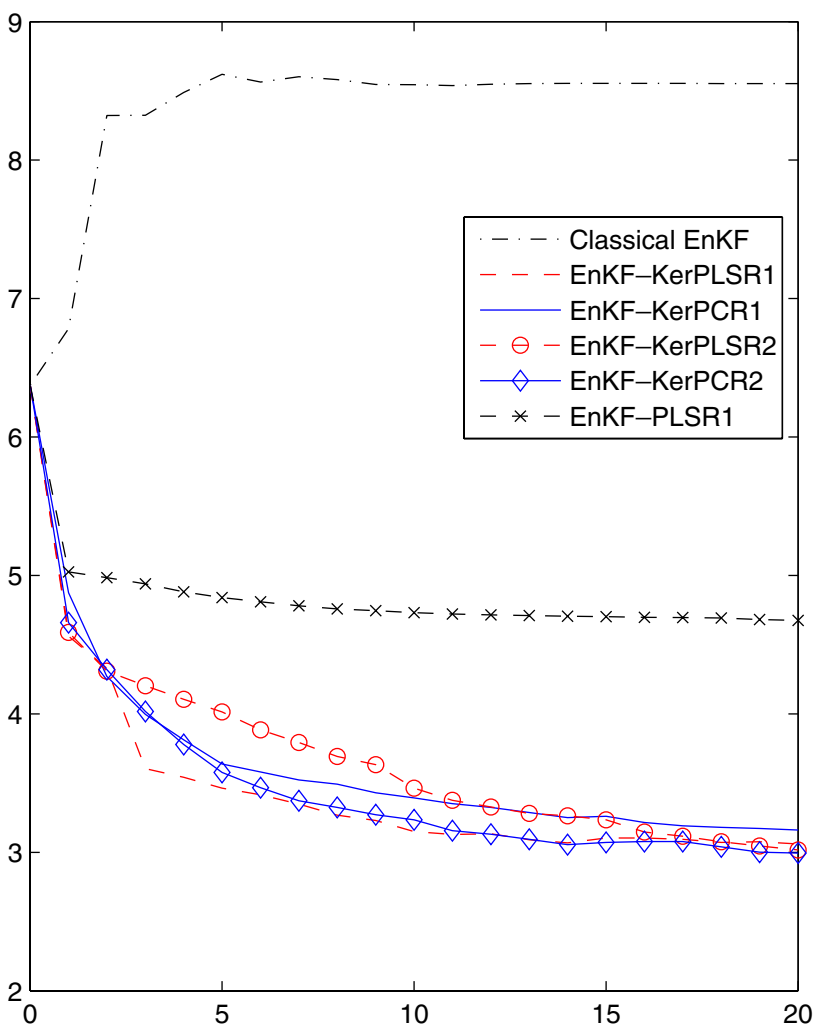

(a) RSS

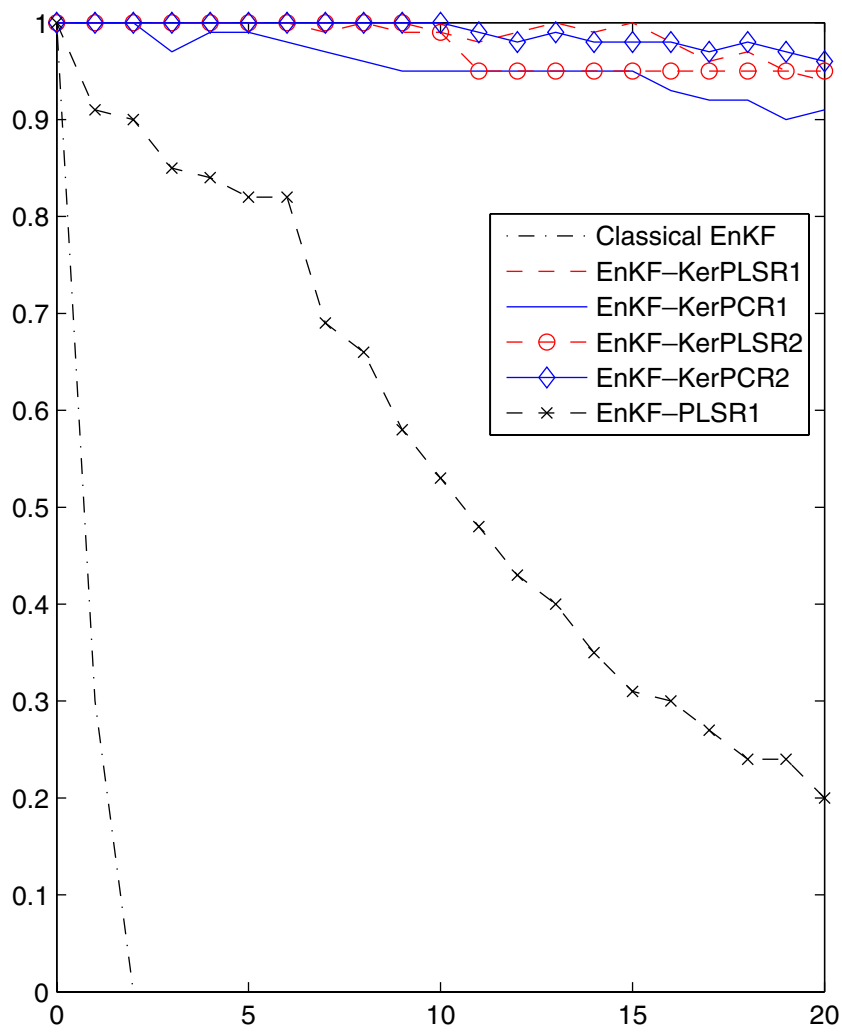

(b) Coverage

Fig. 11 Scaled $\left(1 / n_{e}\right)$ residual sum of squares $(R S S)$, for the forecasted state vector ensemble members and the reference solution, and coverage of the reference solution in the estimated $95 \%$ prediction intervals as a function of time steps $k$ 
selecting the subspace dimension $p$ using 10 -fold CV (EnKF-PLSR1). As we can see from this table, the TSS increase with $412 \%$ from the initial updating step when using the classical EnKF updating scheme. On average, the prediction interval only covers $6.2 \%$ of the reference state vector. Note, however, that the coverage for the majority of the reruns is zero percent.

Using the kernelised shrinkage regression techniques reduces the TSS with between $87 \%$ and $91 \%$, with the largest decrease when we use the supervised parameter selection scheme. This suggests that the PRESS statistic is not the optimal measure of the goodness-of-fit for this model. Replacing the PRESS statistic with alternative measures, is a topic for future research. The estimated mean coverage is close to the theoretical value of $95 \%$ for all four schemes.

Contrary to the classical EnKF updating scheme, using the EnKF-PLSR1 scheme does not lead to an increase in the TSS compared with the initial ensemble. As explained in Sætrom and Omre [46], we expect to see this behaviour because we reduce the problem of regression model overfitting caused by collinear ensemble members, when using shrinkage regression techniques. However, the estimated prediction interval is not able to capture the reference state vector, which we expect when using a linear updating scheme on a highly non-linear likelihood model.

Figure 11a, b, further illustrates the negative effect of model overfitting. Here, the scaled residual sum of squares (RSS) between the ensemble members and the reference solution, $1 / n_{e} \sum_{i=1}^{n_{e}}\left\|\boldsymbol{x}^{c(i)}-\boldsymbol{x}^{\text {True }}\right\|_{2}^{2}$, and the coverage as a function of $k=0, \ldots, 19$ are displayed. As we can see from these figures, the RSS for the classical EnKF is increasing rapidly for the first two updating steps. At the same time, the coverage is rapidly decreasing and after two assimilation steps it is down to zero percent. A similar trend can be seen for the EnKFPLSR1 updating scheme. However, the effect of model overfitting is not as prominent as for the classical EnKF. This illustrates the usefulness of applying shrinkage regression techniques in an EnKF updating scheme.

For the kernelised shrinkage regression techniques, the behaviour of the RSS and coverage is similar for all of the four updating schemes considered. Initially, the RSS decrease rapidly before it stabilises at the later time steps. Similarly, the coverage is slowly converging towards the expected value of $95 \%$, where it appears to stabilise. In addition, note that we are better able to preserve the spread of the updated ensemble members using the kernel-shrinkage regression techniques compared with the EnKF-PLSR1 updating scheme. We believe that the additional non-linearities introduced when mapping the data vector into the feature space is the cause of this behaviour. Hence, we reduce the collinearities between the updated ensemble members, which potentially can lead to model overfitting.

\section{Conclusions}

We have formulated an EnKF updating scheme based on kernel-shrinkage regression techniques to handle highly non-linear and non-Gaussian likelihood models. Contrary to previously suggested EnKF updating schemes based on kernel methods, the approach does not require solving a pre-image problem. Moreover, the computational complexity is equal to the fastest EnKF algorithms previously suggested.

We presented kernel regression as a natural extension of the classical EnKF to the non-linear case using GRF. Under the assumption that the response variable is a GRF, we obtained a prediction scheme defined through the inner product between data vectors in the feature space. We can equally describe these inner products through symmetric, positive definite kernel functions in the Euclidean space, which corresponds to covariance functions for a GRF. Hence, we gave an extension of the Bayesian formulation of the classical EnKF to a non-linear setting, from which kernel RR is a special case. In addition, we considered two additional kernel shrinkage regression techniques based on dimension reduction, namely kernel PCR and PLSR.

We evaluated the performance of the three kernelshrinkage regression techniques for a non-linear regression problem. Here we used a CV scheme to select the prior hyperparameters of the regression models. When we applied the estimated models for prediction purposes, we obtained similar results for the kernel PCR and PLSR methods. However, the dimension of the respective reduced order models was smaller for the kernel PLSR, because this method uses both the predictor and response variables in the dimension reduction. Kernel RR gave slightly larger errors in the predictions.

EnKF updating schemes based on kernel PCR and PLSR were further tested on a hidden Markov model with a non-linear, non-Gaussian likelihood model. For comparison, we considered the standard EnKF updating scheme for non-linear likelihood models. The kernelised shrinkage regression techniques provided good estimates of unknown reference state vector, with uncertainty estimates close to the theoretical bounds. On this model, the updating scheme based on kernel PCR performed slightly better than kernel PLSR, although the subspace dimension tended to be smaller when using the supervised PLSR approach. The standard EnKF, on the other hand, completely missed the 
reference solution, with an ensemble collapsing after a few updating steps.

Acknowledgement This work is funded by the Uncertainty in Reservoir Evaluation (URE) consortium at NTNU.

Open Access This article is distributed under the terms of the Creative Commons Attribution Noncommercial License which permits any noncommercial use, distribution, and reproduction in any medium, provided the original author(s) and source are credited.

\section{Appendix A Gaussian random field}

The collection of random variables $\left\{r\left(\boldsymbol{x}_{1}\right), \ldots, r\left(\boldsymbol{x}_{n_{e}}\right)\right\}$ is a Gaussian random field (GRF) if any subset of the random variables $\left\{r\left(\boldsymbol{x}_{i}\right)\right\}$ has a joint Gaussian distribution. A GRF is completely specified through a mean and covariance function, denoted $m(\boldsymbol{x})$, and $c\left(\boldsymbol{x}, \boldsymbol{x}^{\prime}\right)$ respectively, for any vectors $\boldsymbol{x}$ and $\boldsymbol{x}^{\prime} \in \mathbb{R}^{n_{x} \times 1}$. Here, $m(\boldsymbol{x})=\mathrm{E}[r(\boldsymbol{x})]]$ and

$c\left(\boldsymbol{x}, \boldsymbol{x}^{\prime}\right)=\mathrm{E}\left[(r(\boldsymbol{x})-m(\boldsymbol{x}))\left(r\left(\boldsymbol{x}^{\prime}\right)-m\left(\boldsymbol{x}^{\prime}\right)\right)^{T}\right]$

Whilst $m: \mathbb{R}^{n_{x}} \rightarrow \mathbb{R}$ can be any function, the following criteria must be satisfied for the covariance function $c:\left(\mathbb{R}^{n_{x} \times 1} \times \mathbb{R}^{n_{x} \times 1}\right) \rightarrow \mathbb{R}:$

1. Symmetric: $c\left(\boldsymbol{x}, \boldsymbol{x}^{\prime}\right)=c\left(\boldsymbol{x}^{\prime}, \boldsymbol{x}\right)$.

2. Positive semidefinite:

$$
\int c\left(\boldsymbol{x}, \boldsymbol{x}^{\prime}\right) f(\boldsymbol{x}) f\left(\boldsymbol{x}^{\prime}\right) \mathrm{d} \mu(\boldsymbol{x}) \mathrm{d} \mu\left(\boldsymbol{x}^{\prime}\right) \geq 0,
$$

$$
\text { for all } \boldsymbol{x} \text { and } \boldsymbol{x}^{\prime} \in \mathbb{R}^{n_{x} \times 1} \text { and } f \in L_{2}\left(\mathbb{R}^{n_{x}}, \mu\right) \text {. }
$$

Within the class of valid covariance functions are the stationary and isotropic:

$c\left(\boldsymbol{x}, \boldsymbol{x}^{\prime}\right)=c\left(\left\|\boldsymbol{x}-\boldsymbol{x}^{\prime}\right\|_{2}\right)$,

where $\|\cdot\|_{2}$ is the Euclidean norm.

\section{Appendix B Computational properties}

If the model parameters, $\boldsymbol{\theta}$ are specified, the computational complexity of the EnKF updating schemes based on kernel RR, kernel PCR and kernel PLSR is $\mathcal{O}\left(\max \left\{n_{d}, n_{x}, n_{e}\right\} n_{e}^{2}\right)$. Hence, we have the same computational complexity and memory requirements as the fastest EnKF algorithms previously suggested when $n_{e}<\max \left\{n_{x}, n_{d}\right\}$. Further note that we can write the updating scheme based on kernel RR, similarly to kernel PCR, using the $n_{e}$ estimated eigenvectors and values of the centred kernel matrix. This follows because $\boldsymbol{H C H}$ by construction is symmetric, positive semidefinite.
When the model parameters, $\boldsymbol{\theta}$ are selected based on a CV scheme, as outlined in Algorithm 1, the computational complexity of the respective kernelised EnKF updating schemes is $\mathcal{O}\left(r \max \left\{n_{x}, n_{d}, n_{e}\right\} n_{e}^{2}\right)$. In the example considered in this paper, $r=n_{\sigma} n_{e}$, where $n_{\sigma}$ denotes the number of discrete values of $\sigma$ we consider in the Gaussian kernel function, which in this study is $n_{e} / 4$. Hence, in terms of the computational complexity, it is preferable to pre-select the model parameters because the computational demands decrease by a factor $n_{e}^{2}$. However, in the general case we expect that using pre-selected model parameters will be a less robust choice, compared with the alternative of selecting the model parameters at each updating step using CV.

\section{References}

1. Aanonsen, S.I., Nævdal, G., Oliver, D.S., Reynolds, A.C., Vallès, B.: Ensemble Kalman filter in reservoir engineeringa review. SPE J. 14(3), 393-412 (2009)

2. Anderson, J.L.: A local least squares framework for ensemble filtering. Mon. Weather Rev. 131(4), 634-642 (2003)

3. Caers, J., Park, K.: Distance-based representation of reservoir uncertainty: the metric EnKF. In: Proceeding to the ECMOR XI, Sept 8-11. Bergen, Norway (2008)

4. Casella, G., Berger, R.L.: Statistical Inference. Duxbury (2002)

5. Chiles, J.-P., Delfiner, P.: Geostatistics, Modeling Spatial uncertainty. Wiley, New York (1999)

6. Doucet, A., Godsill, S., Andrieu, C.: On sequential Monte Carlo sampling methods for Bayesian filtering. Stat. Comput. 10(3), 197-208 (2000)

7. Evensen, G.: Sequential data assimilation with nonlinear quasi-geostrophic model using Monte Carlo methods to forecast error statistics. J. Geophys. Res. 99, 10143-10162 (1994)

8. Evensen, G.: Data Assimilation. The ensemble Kalman filter. Springer, New York (2007)

9. Farrer, D.E., Glauber, R.R.: Multicollinearity in regression analysis: the problem revisited. Rev. Econ. Stat. 49(1), 92-107 (1967)

10. Furrer, R., Bengtsson, T.: Estimation of high-dimensional prior and posterior covariance matrices in Kalman filter variants. J Multivariate Anal. 98(2), 227-255 (2007)

11. Gibbs, M.N.: Bayesian Gaussian Processes for Regression and Classification. Ph.D. thesis, University of Cambridge (1997)

12. Hastie, T., Tibshirani, R., Freidman, J.: The Elements of Statistical Learning; Data Mining, Inference, and Prediction, 2nd edn. Springer, New York (2009)

13. Helland, I.S.: Some theoretical aspects of partial least squares regression. Chemometr. Intell. Lab. Syst. 58(2), 97-107 (2001)

14. Hoerl, A.E., Kennard, R.W.: Ridge Regression: Biased estimation for nonorthogonal problems. Technometrics 12(3), 55-67 (1970)

15. Hofmann, T., Schölkopf, B., Smola, A.J.: Kernel methods in machine learning. Ann. Stat. 36(3), 1171-1220 (2008) 
16. Hotelling, H.: Analysis of a complex of statistical variables into Principal Components. J. Educ. Psychol. 24(6), 417-441 and 498-520 (1933)

17. Houtekamer, P.L., Mitchell, H.L.: Data assimilation using an ensemble Kalman filter technique. Mon. Weather Rev. 126, 796-811 (1998)

18. Huang, T.M., Kecman, V., Kopriva, I.: Kernel based algorithms for mining huge data sets, supervised, semi-supervised, and unsupervised learning. Springer, New York (2006)

19. Journel, A.G., Huijbregts, C.J.: Mining Geostatistics. Academic, London (1978)

20. Kalivas, J.H.: Cyclic subspace regression with analysis of the hat matrix. Chemometr. Intell. Lab. Syst. 45, 215-224 (1999)

21. Kalman, R.E.: A new approach to linear filtering and prediction problems. Trans ASME J Basic Eng 82(Series D), 35-45 (1960)

22. Kennedy, J., Eberhart, R.: Particle swarm optimization. In: Proceedings of the IEEE International Conference on Neural Networks, Piscataway, NJ, pp. 1942-1948 (1995)

23. Kirkpatrick, S., Gelatt, C.D., Vecchi, M.P.: Optimization by simulated annealing Science 220(4598), 671-680 (1983)

24. Kwok, J.T., Tsang, I.W.: The pre-image problem in kernel methods. IEEE Trans. Neural Netw. 15(6), 1517-1525 (2004)

25. Mardia, K.V., Kent, J.T., Bibby, J.M.: Multivariate analysis. Academic, London (1979)

26. Mika, S., Schölkopf, B., Smola, A., Müller, R., Scholz, M., Rätch, G.: Advances in neural information processing systems 11, chapter kernel PCA and de-noising in feature spaces. Morgan Kaufmann, San Mateo (1998)

27. Myrseth, I.B., Omre, H.: Large-scale inverse problems and quantification of uncertainty, chapter the ensemble kalman filter and related filters. John Wiley and Sons (2010)

28. Myrseth, I.B., Sætrom, J., Omre, H.: Resampling the ensemble Kalman filter. Paper submitted for publication (2010)

29. Nocedal, J., Wright, S.: Numerical optimization. Springer, New York (2006)

30. O'Hagan, A.: Curve fitting and optimal design for prediction. J. R. Stat. Soc. B (Met.) 40(1), 1-42 (1978)

31. Oliver, D.: On conditional simulation to inaccurate data. Journal of Mathematical Geology 28(6), 811-817 (1996)

32. Rao, C.R.: Linear Statistical Inference and Its Applications, 2nd edn. Wiley, New York (1973)

33. Rasmussen, C.E., Williams, C.K.I.: Gaussian Processes for Machine Learning. MIT Press (2006)

34. Ränner, S., Lindgren, F., Gelandi, P., Wold, S.: A PLS kernel algorithm for data sets with many variables and fewer objects. Part 1: Theory and Algorithm. J. Chemometr. 8(2), 111-125 (1994)
35. Rosipal, R., Girolami, M., Trejo, L.J., Cichocki, A.: Kernel PCA for feature extraction and de-noising in non-Linear regression. Neural Comput Appl 10(3), 231-243 (2001)

36. Rosipal, R., Trejo, L.J.: Kernel Partial Least Squares regression in reproducing kernel Hilbert space. J Mach Learn Res 2, 97-123 (2002)

37. Sacher, W., Bartello, P.: Sampling errors in Ensemble Kalman filtering. Part I: Theory. Mon. Weather Rev. 136(8), 3035-3049 (2008)

38. Sarma, P., Chen, W.H.: Generalization of the Ensemble Kalman Filter using kernels for nongaussian Random Fields. In: Proceedings to the Reservoir Simulation Symposium, SPE, The Woodlands, Texas, 2-4 February 2009 (2009)

39. Sarma, P., Durlofsky, L., Aziz, K.: Kernel principal component analysis for an efficient, differentiable parametrization of multipoint geostatistics. Math. Geol. 40(1), 3-32 (2008)

40. Scales, J.A., Snieder, R.: To Bayes or not to Bayes? Geophysics 62(4), 1045-1046 (1997)

41. Schölkopf, B., Smola, A., Muller, K.: Nonlinear component analysis as a kernel eigenvalue problem. Neural Comput. 10(5), 1299-1319 (1998)

42. Seber, G.A.F., Lee, A.J.: Linear Regression Analysis. Wiley, New York (2003)

43. Smola, A.: Learning with Kernels. Ph.D. thesis, TU Berlin (1998)

44. Smola, A., Schölkopf, B.: Learning with Kernels. Springer, New York (2002)

45. Smola, A., Schölkopf, B., Müller, K.: The connection between regularization operators and support vector kernels. Neural Networks 11(4), 637-649 (1998)

46. Sætrom, J., Omre, H.: Ensemble Kalman filtering with shrinkage regression techniques. Comput. Geosci. 15(2), 271$292(2011)$

47. Taylor, J.S., Cristianini, N.: Kernel methods for pattern analysis. Cambridge University Press, Cambridge (2004)

48. van Leeuwen, P.J.: Comments on "Data assimilation using an ensemble Kalman filter technique". Mon. Weather Rev. 127, 1374-1377 (1999)

49. Vapnik, V.: Statistical Learning Theory. Wiley, New York (1998)

50. Williams, C.K.I.: Learning and inference in graphical models, chapter prediction with Gaussian processes: from linear regression to linear prediction and beyond. Kluwer, Norwell (1998)

51. Wold, H.: Quantitative sociology: international perspectives on mathematical and statistical model building, chapter path models with latent variables: the NiPALS Approach. pp. 307 357. Academic, London (1975)

52. Young, N.: An introduction to Hilbert Space. Cambridge University Press, Cambridge (1988) 